\title{
Conformal Gauges and Renormalized Equations of Motion in Massless Quantum Electrodynamics
}

\author{
V. B. Petkova, G. M. Sotkov, and I. T. Todorov \\ Institute for Nuclear Research and Nuclear Energy, \\ Bulgarian Academy of Sciences, BG-1184 Sofia, Bulgaria
}

To the memory of Kurt Symanzik

\begin{abstract}
A formulation of massless QED is studied with a non-singular Lagrangian and conformal invariant equations of motion. It makes use of nondecomposable representations of the conformal group $G$ and involves two dimensionless scalar fields (in addition to the conventional charged field and electromagnetic potential) but gauge invariant Green functions are shown to coincide with those of standard (massless) QED. Assuming that the (nonelementary) representation of $G$ for the 5-potential which leaves the equations of motion invariant and leads to the free photon propagator of Johnson-BakerAdler (JBA) conformal QED remains unaltered by renormalization, we prove that consistency requirements for conformal invariant 2-, 3-, and 4-point Green functions satisfying (renormalized) equations of motion and standard Ward identities lead to either a trivial solution (with $e \psi=0$ ) or to a subcanonical dimension $d=\frac{1}{2}$ for the charged field.
\end{abstract}

\section{Introduction}

The search for a conformal invariant quantum field theory (QFT) is one way to look for a (critical) renormalization group fixed point (see, e.g., [S2] where the essential equivalence between the two problems has been spelled out). It is, therefore, intimately related to the existence problem for a local relativistic QFT (see [A3, F5, M5]).

The study of conformal quantum electrodynamics (QED) [J1, A1, 2, E1, M4, F6, B1] (see also Chap. VII to [T1]) differs in at least two points from a parallel investigation of a nongauge, Yukawa-type QFT (see [M2, 3, D3, 4, F4, T1] and references therein). First, current conservation and the Maxwell equations imply that the dimension of one of the basic fields, the 4-potential $A_{\mu}(x)$, is canonical (while the dimension of the charged field $\Psi(x)$ is gauge dependent). Secondly, although conformal invariance of the classical (vacuum) Maxwell equations has been known since the time when application of group theory to physics was a novelty (see [C1, B2]), the problem of finding a conformal invariant gauge fixing 
term has been treated only recently (starting with [M7, B3]. The non-invariance of conventional (local) gauges poses problems in exploiting conformal symmetry for gauge dependent Green functions like the photon propagator.

It was gradually realized that the electromagnetic potential should transform under a non-elementary representation of the conformal group $G=\operatorname{SU}(2,2)$ (extended by space reflections) or its Euclidean counterpart $\operatorname{Spin}(5,1)$ (otherwise the invariant photon propagator comes out purely longitudinal). The most attractive approach, in our view, uses non-decomposable representations of $G$ and leads to a 5-potential (see the evolution of these ideas in ${ }^{1}$ [S3, B4, Z1, F7]). Alternatively, direct sums of irreducible subrepresentations and factor representations of exceptional elementary representations for $A_{\mu}$ have also been tried ([F3, P2]).

The present paper studies possible conformal invariant solutions of the model of massless QED, put forward in [F7]. We prove that if renormalization does not change the transformation law for the 5-potential (which leaves the classical equations of motion invariant and leads to a free photon propagator as in the JBA approach), then the only conformal solution of the model corresponds to the vanishing of a product of the type $e C_{\psi} Z(d) \frac{\Gamma\left(d+\frac{1}{2}\right)}{\Gamma\left(\frac{5}{2}-d\right)}$, where $e$ is the electric charge, $C_{\psi}$ is a normalization constant of the (renormalized) electron propagator, and $Z(d)$ is the finite part of the electron field renormalization constant $Z_{2}, d$ being the scale dimension of the (interacting) electron field. From the analysis of the (renormalized) Schwinger-Dyson form of the Dirac equation and the small distance behaviour of the 3-point Schwinger function $\left\langle A_{\mu}\left(x_{1}\right) \Psi\left(x_{2}\right) \Psi\left(x_{3}\right)\right\rangle_{E}$ we deduce (in Sect. 4) that this consistency condition amounts to

$$
e\left(\frac{7}{2}-d\right)\left(\frac{5}{2}-d\right)\left(d-\frac{1}{2}\right)=0 .
$$

It will be made clear in Sect. 3 that if we do not allow a logarithmic behaviour for the Euclidean 2-point function of $\psi$, then its conformal invariant Wightman function vanishes for $d-\frac{5}{2}=0,1, \ldots$ Thus Eq. (1.1) means that either $e=0$ (a result asserted in [K1] for the model of [F3,P2]) or $\psi=0$, or else the (gauge dependent!) scale dimension $d$ takes the subcanonical value $d=\frac{1}{2}$. Thus finite conformal electrodynamics with a free (JBA) photon propagator is either trivial or only exists for a particular class of gauges that imply $d=\frac{1}{2}$ for $e \neq 0$. Strictly speaking, we only verify that the Schwinger-Dyson equations and Ward identities involving 2-, 3-, and some 4-point functions are consistent with the conformal postulate provided that Eq. (1.1) is satisfied. The existence proof for a non-trivial model for $d=\frac{1}{2}$ is a challenging open problem. In any case, it is already clear that a more careful argument is needed to justify recent claims [K1, M6] that finite charge renormalization in QED is impossible. We also point out that conformal invariance does not necessarily lead to free photon propagators. The possibility of using more complicated operator transformation laws (including nondecomposable representations of the subgroup of dilations [D1, F1] that lead, in

1 The last three papers contain essentially equivalent sets of equations for a 5-potential interacting with a conserved (external) current. However, it is only in [F7] that a non-singular conformal action is proposed which also includes charged (spinor) fields 
particular, to a logarithmic term in the transverse part of the photon propagator) has not been fully explored.

We start in Sect. 2 with a new concise derivation of the equations of motion (and the Lagrangian) of the model within the classical framework. We give a formal argument in Sect. 2C (based on Feynman path integration) that in spite of the presence of non-conventional terms in the equations involving two dimensionless scalar fields the gauge invariant Green functions coincide (at least within the framework of perturbation theory) with those of standard massless QED. Section 3 is devoted to the "conformal quantization" of the model (in which some of the canonical commutation relations are traded for the assumption that renormalized Green functions are conformal invariant). Ward identities are studied in detail. It is demonstrated that they imply a deformation of the charged field transformation law (with deformation parameter $e$ ). Section 4 is devoted to the renormalization program. The discussion is based on combined use of conformal invariance and Wilson (operator product) expansions (together with a systematic application of Euclidean symmetric integration) see [S6, P1, D5].

\section{Notational Conventions}

Minkowski space metric: $x p=x^{\mu} \eta_{\mu \nu} p^{\nu}=\mathbf{x p}-x^{0} p^{0} ;\left[\gamma_{\mu}, \gamma_{v}\right]_{+}=2 \eta_{\mu \nu}$ (the physical time variable is $t=x^{0}$, the energy is $E=-p_{0}$ ).

Euclidean (Schwinger) functions and their Fourier transforms. Scalar field of dimension $\delta$ :

$$
\Delta_{\delta}(x)=\frac{1}{(4 \pi)^{2}} \frac{\Gamma(\delta)}{\Gamma(2-\delta)}\left(\frac{4}{x^{2}}\right)^{\delta}=\int \frac{e^{i p x}}{\left(p^{2}\right)^{2-\delta}} d_{4} p,
$$

Spinor field $\psi$ of dimension d:

$$
\begin{gathered}
S_{d}=\frac{1}{C_{\psi}}\langle\psi(x) \bar{\psi}(0)\rangle_{E}=\frac{1}{(4 \pi)^{2}} \frac{\Gamma\left(d+\frac{1}{2}\right)}{\Gamma\left(\frac{5}{2}-d\right)}\left(\frac{4}{x^{2}}\right)^{d+1 / 2} \frac{\not x}{2} \\
=-i \int \frac{\not p e^{i p x}}{\left(p^{2}\right)^{5 / 2-d}} d_{4} p,\left(S_{\psi}(x)=C_{\psi} S_{d}(x)\right), \not p=\gamma^{\mu} p_{\mu} \\
d_{4} p=\frac{d^{4} p}{(2 \pi)^{4}}, \quad x^{2}=\mathbf{x}^{2}+x_{4}^{2}, \quad x_{4}=-i x^{0}, \\
\gamma_{4}=i \gamma_{0}, \quad\left(C_{\psi}=C_{\psi}(d), C_{\psi}\left(\frac{3}{2}\right)=1\right) .
\end{gathered}
$$

\section{Conformal Electrodynamics Involving a Non-Decomposable 5-Potential}

A) Nonsingular Conformal Lagrangian for the Electromagnetic Potential from a Manifestly Covariant Connection Form

The most natural way to introduce a non-trivial conformal 5-potential is to use Dirac's manifestly covariant formalism [D2, M1]. 
Compactified Minkowski space $\bar{M}$ is realized as the projective quadric:

$$
\begin{gathered}
\bar{M}=Q / \mathbb{R}^{*}, \quad \mathbb{R}^{*}=\mathbb{R} \backslash\{0\}, \\
Q=\left\{\xi=\left(\xi^{0}, \xi, \xi^{5}, \xi^{6}\right) \in \mathbb{R}^{6} ;\right. \\
\left.\xi^{2}=\xi^{a} \eta_{a b} \xi^{b}=-\xi_{0}^{2}+\xi^{2}+\xi_{5}^{2}-\xi_{6}^{2}=0, \xi \neq 0\right\} .
\end{gathered}
$$

Identifying the points at infinity with the projective 3-cone

$$
I_{\infty}=Q_{\infty} / \mathbb{R}^{*}, \quad Q_{\infty}=\left\{\xi \in Q ; \kappa \equiv \xi^{5}+\xi^{6}=0,\left(=\xi_{\mu} \xi^{\mu}\right)\right\},
$$

we imbed (real) Minkowski space $M$ into $\bar{M}$ by setting

$$
\begin{gathered}
(M \ni) x^{\mu}=\frac{1}{\kappa} \xi^{\mu}, \quad \mu=0,1,2,3, \quad \text { for } \quad \kappa\left(=\xi^{5}+\xi^{6}\right) \neq 0 ; \\
\text { thus } \quad M \simeq \bar{M} \backslash I_{\infty} .
\end{gathered}
$$

The 5-potential is defined as a homogeneous (in $\xi$ ) real valued 1 -form on the quadric $Q$ :

$$
\mathscr{A}_{a}(\xi) d \xi^{a}\left(=\mathscr{A}_{a}(\varrho \xi) d \varrho \xi^{a}\right) \text { for } \quad \xi_{a} d \xi^{a}=0\left(=\xi_{a} \xi^{a}\right) .
$$

In the local coordinates $(x, \kappa)$ on $Q \backslash Q_{\infty}$ we can write

$$
\mathscr{A}_{a}(\xi) d \xi^{a}=A_{\mu}(x) d x^{\mu}+A_{-}(x) \frac{d \kappa}{\kappa},
$$

where $\mathscr{A}_{\mu}(x)=\kappa \mathscr{A}_{\mu}(\xi)+\left(\mathscr{A}_{6}(\xi)-\mathscr{A}_{5}(\xi)\right) \xi_{\mu} \quad$ and $A_{-}(x)=\xi^{a} \mathscr{A}_{a}(\xi)$ are $\quad \kappa-$ independent Poincaré covariant vector and scalar fields with the following transformation properties under dilations $x \rightarrow \varrho x, \varrho>0$ and (infinitesimal) special conformal transformations

$$
\begin{gathered}
x^{\mu} \rightarrow^{\prime} x^{\mu}=\omega\left(x^{\mu}+x^{2} c^{\mu}\right)\left(\approx x^{\mu}(1-2 c \cdot x)+x^{2} c^{\mu} \quad \text { for } \quad c^{\mu} \rightarrow 0\right), \\
\omega^{-1}=1+2 c x+c^{2} x^{2}, \\
A_{-}(x) \rightarrow A_{-}(\varrho x), \quad A_{v}(x) \rightarrow \varrho A_{v}(\varrho x) \\
A_{N}(x) \rightarrow A_{N}(x)+c^{\mu}\left[C_{\mu}, A_{N}(x)\right]
\end{gathered}
$$

where $N=v,-$ and

$$
\begin{aligned}
{\left[A_{-}(x), C_{\mu}\right]=\left(2 x_{\mu} x \partial-x^{2} \partial_{\mu}\right) A_{-}(x), } \\
{\left[A_{v}(x), C_{\mu}\right]=\left[2 x_{\mu}(1+x \partial)-x^{2} \partial_{\mu}\right] A_{v}(x) } \\
+2\left(\eta_{\mu v} x A(x)-x_{v} A_{\mu}(x)-\eta_{\mu v} A_{-}(x)\right) .
\end{aligned}
$$

The crucial property is the appearance of $A_{-}$in the right-hand side of $(2.4 \mathrm{~b})$, which is responsible for the non-vanishing of $\left.\left[A_{v}(x), C_{\mu}\right]\right|_{x=0}$. As a consequence the Maxwell tensor $F_{\mu \nu}=\partial_{\mu} A_{\nu}-\partial_{\nu} A_{\mu}$ is also transformed under a non-elementary non-decomposable representation of the (local) conformal group, together with 
$\partial_{v} A_{-} ;$we have

$$
\begin{aligned}
{\left[\partial_{\mu} A_{-}(x), C_{\lambda}\right]=} & {\left[2 x_{\lambda}(1+x \partial)-x^{2} \partial_{\lambda}\right] \partial_{\mu} A_{-}(x) } \\
& +2\left(\eta_{\lambda \mu} x \partial-x_{\mu} \partial_{\lambda}\right) A_{-}(x) \\
{\left[F_{\mu \nu}(x), C_{\lambda}\right]=} & {\left[2 x_{\lambda}(2+x \partial)-x^{2} \partial_{\lambda}\right] F_{\mu \nu}(x) } \\
& +2\left(x^{\kappa} \eta_{\lambda[\mu} F_{\kappa \nu]}-x_{[\mu} F_{\lambda \nu]}+\eta_{\lambda[\mu} \partial_{\nu]} A_{-}\right),
\end{aligned}
$$

where $\eta_{\lambda[\mu} F_{\kappa \nu]} \equiv \eta_{\lambda \mu} F_{\kappa \nu}-\eta_{\lambda \nu} F_{\kappa \mu}$, etc.

Taking the divergence $\partial^{v}$ of both sides of (2.6) and the d'Alembertian of (2.5), we deduce that only the combination

$$
J^{\mu}=\partial_{\nu} F^{\mu \nu}-\frac{1}{2} \square \partial^{\mu} A_{-}
$$

transforms as a conformal 4-vector. Therefore, if $J^{\mu}$ is identified with the electromagnetic current of a charged field, setting, e.g.,

$$
J^{\mu}=i e \bar{\psi} \gamma^{\mu} \psi,
$$

then Eq. (2.7) can be regarded as a conformal extension of the standard Maxwell equation. On the other hand, applying $\square \partial^{v}$ to both sides of $(2.4 \mathrm{~b})$, we obtain

$$
\left[\square \partial A, C_{\mu}\right]=\left[2 x_{\mu}(3+x \partial)-x^{2} \partial_{\mu}\right] \square \partial A+2 \partial^{v} F_{\mu \nu}-\square \partial_{\mu} A_{-} .
$$

Hence, the equation

$$
\frac{1}{2} \square \partial A=J_{+}
$$

can be regarded as a covariant complement of the conformal Maxwell equation (2.7) provided that $\left(J^{\mu}, J_{+}\right)$transforms as a 5-current; in particular,

$$
\left[\left(\begin{array}{c}
J_{+} \\
J^{\mu}
\end{array}\right), C_{\lambda}\right]_{x=0}=\left(\begin{array}{c}
2 J_{\lambda}(0) \\
0
\end{array}\right),
$$

so that we can not use the simple conformal gauge condition $\square \partial A=0$ (of refs. [M2, B3]) in the interacting case.

It follows from the properties of conformal representation spaces (see [P3]) that the integral

$$
\int J_{+}(x) d^{4} x
$$

is convergent and, according to (2.10) and Gauss theorem, it is expressed in terms of the asymptotic values of $\square A_{\mu}$ at infinity. Current conservation

$$
\partial^{\mu} J_{\mu}=0
$$

guarantees its conformal invariance. As we shall see (in Sect. 2.C below), it is also gauge invariant.

Assuming that $\left(J^{N}\right)=\left(J^{v}, J_{+}\right)$is a given conserved external 5-current, we can write the following canonical Lagrangian for the 5-potential $A_{N}$ which yields the conformal invariant equations of motion (2.7), (2.10), and (2.13):

$$
\begin{aligned}
L(A, J)= & \frac{1}{2} F^{\mu \nu}\left(\frac{1}{2} F_{\mu \nu}-\partial_{\mu} A_{v}+\partial_{\nu} A_{\mu}\right)-\frac{1}{2} A_{-} \square \partial A \\
& +\frac{\beta}{8}\left(\square A_{-}\right)^{2}+J_{+} A_{-}+J^{\mu} A_{\mu} .
\end{aligned}
$$


It is non-singular; in particular, each independent field variable $A_{\mu}$ and $A_{-}$has a non-vanishing conjugate momentum:

$$
\pi^{\mu}=\frac{\partial L}{\partial \partial_{0} A_{\mu}}=-F^{0 \mu}+\frac{1}{2} \delta_{0}^{\mu} \square A_{-}, \quad \pi_{-}=-\frac{1}{2} \partial_{0} \partial A+\frac{\beta}{8} \partial_{0} \square A_{-} .
$$

The results of this section can be summarized as follows.

Proposition 2.1. If the electromagnetic 5-potential $A_{N}=\left(A_{v}, A_{-}\right)$obeys the nondecomposable conformal transformation law (2.3) (2.4) (derived from the invariance of the homogeneous connection form (2.1) on the 5-dimensional quadric $Q$ ), then the Lagrangian (2.14) gives rise to the conformal invariant field equations

$$
\mathscr{M}(\partial)\left(A^{N}\right) \equiv\left(\begin{array}{ll}
\partial^{\mu} \partial_{v} \square & -\frac{1}{2} \partial^{\mu} \square \\
\frac{1}{2} \partial_{v} \square & -\frac{1}{4} \beta \square^{2}
\end{array}\right)\left(\begin{array}{c}
A^{v}(x) \\
A_{-}(x)
\end{array}\right)=\left(\begin{array}{c}
J^{\mu}(x) \\
J_{+}(x)
\end{array}\right)
$$

that describe the interaction of a conserved (external) (5-)current with $A_{N}$. Current conservation (2.13) implies the generalized free field equation

$$
\square^{2} A_{-}=0,
$$

which yields $\beta$-independence of the equations. The Lagrangian is non-singular, the (Euclidean) $p$-space matrix $\mathscr{M}(i p)$ being invertible for $p \neq 0$ whatever the value of the gauge parameter $\beta .\left(\mathscr{M}^{-1}(i p)\right.$ will be identified with the propagator matrix in Sect.3B.)

\section{B) Construction of $J_{+}$. Conformal Dirac Equation}

The fifth component $J_{+}$of the 5-current cannot be expressed in terms of a conventional (charged) Dirac field $\Psi$ only. An economic way to construct $J_{+}$, proposed in [S3, F7] uses a longitudinal 5-potential coming from the closed form $d S(\xi)=\partial_{\mu} S(x) d x^{\mu}+q \frac{d \kappa}{\kappa}$, where $q$ is a constant. $\left(\partial_{\mu} S, q\right)$ spans an invariant subspace of the non-decomposable space of 5-potentials $\left(A_{\mu}, A_{-}\right)$(obeying the same transformation law (2.3), (2.4) which could be also deduced from the following law for the dimensionless scalar field $S(x)$ :

$$
\begin{gathered}
S(x) \rightarrow S(\varrho x)-q \ln \varrho ; \\
{\left[S(x), C_{\mu}\right]=2 x_{\mu}(x \partial S(x)-q)-x^{2} \partial_{\mu} S(x)}
\end{gathered}
$$

see $[\mathrm{S} 1, \mathrm{D} 1, \mathrm{~F} 1])$.

Proposition 2.2. If $j^{\mu}$ is a conserved conformal vector current and S obeys (2.16), then

$$
J^{\mu}=q j^{\mu} \quad \text { and } \quad J_{+}=-j^{v} \partial_{v} S+\alpha_{1} j^{v} \partial_{v} A_{-}+\alpha_{2} \square^{2} S
$$

transform as a 5-current for any values of the (real) parameters $\alpha_{1}$ and $\alpha_{2}$.

Proof. Equation (2.16) and the infinitesimal conformal transformation law for $j^{v}$ imply $\left[J_{+}(x), C_{\mu}\right]=\left(2 x_{\mu}(4+x \partial)-x^{2} \partial_{\mu}\right) J_{+}+2 J_{\mu}(x)$ in accord with (2.11).

In the classical theory we could set $q=1$. We shall see, however, that quantization forces us to regard $q$ as a (constant) dynamical variable. It seems, 
nevertheless, consistent to assume at both the classical and the quantum level that

$$
J^{v}(x)=i e: \bar{\psi}(x) \gamma^{v} \psi(x):=q J^{v}(x) .
$$

We shall also restrict our attention to the choice $\alpha_{1}=0=\alpha_{2}$ in Eq. (2.17), thus having

$$
J_{+}(x)=-J^{v}(x) \partial_{v} S(x) .
$$

Combining (2.14) with (2.8) and (2.18) and adding (massless) conformal invariant kinetic terms for $\psi$ and $S$, we end up with the following Lagrangian for conformal electrodynamics:

$$
L(A, S, \psi, \bar{\psi})=L(A, J)-\frac{1}{2} \bar{\psi} \overleftrightarrow{\phi} \psi+\frac{v}{2}(\square S)^{2}, \quad \not \partial=\partial_{\mu} \gamma^{\mu} .
$$

It reproduces Eqs. (2.7) and (2.10) (with $J^{N}$ expressed in terms of $\psi$ and $S$ ) and implies in addition the (modified) Dirac equation

$$
\left[\not \partial-i e\left(A-A_{-} \not \partial S\right)\right] \Psi=0
$$

and a $4^{\text {th }}$ order equation for $S$

$$
v \square^{2} S=\partial_{\mu}\left(A_{-} J^{\mu}\right)=i e \bar{\psi}\left(\not A_{-}\right) \psi .
$$

In order to verify the conformal invariance of Eq. (2.20), we use the infinitesimal laws (2.4) (2.16) and

$$
\begin{aligned}
{\left[\psi(x), C_{\mu}\right] } & =\left\{2 x_{\mu}\left(\frac{3}{2}+x \partial\right)-x^{2} \partial_{\mu}+\frac{1}{2}\left[\gamma_{\mu}, x\right]\right\} \psi(x), \\
{\left[\partial \psi(x), C_{\mu}\right] } & =\left\{2 x_{\mu}\left(\frac{5}{2}+x \partial\right)-x^{2} \partial_{\mu}+\frac{1}{2}\left[\gamma_{\mu}, x\right]\right\} \not \psi \psi(x) .
\end{aligned}
$$

[They imply, in particular, that $\left(A-A_{-} \not \partial S\right) \psi$ has the same conformal transformation law as $\not \psi$.]

Remark 2.3. Clearly, the Lagrangian (2.19), and hence, the equations of motion are invariant under the discrete $\gamma_{5}$ transformation $\psi \rightarrow i \gamma_{5} \psi, \bar{\psi} \rightarrow i \bar{\psi} \gamma_{5} ;\left(J^{N} \rightarrow J^{N}, A_{N}\right.$ $\rightarrow A_{N}, S \rightarrow S$ ). Moreover, the equations of motion imply that the axial current $J_{5}^{\mu}=i e: \bar{\psi} \gamma_{\mu} \gamma_{5} \psi:$ is also conserved; this allows us to decouple the left and right handed Weyl spinors in the Dirac equation.

\section{C) Gauge Properties. Relation to a Non-Local Gauge Fixing}

The action integral $\int L d^{4} x$, and hence the entire set of Eqs. (2.18), (2.15), (2.20), (2.21) are invariant under the (restricted) gauge transformations

$$
\begin{gathered}
\psi(x) \rightarrow e^{i e(\Lambda(x)+b S(x))} \Psi(x)\left(\bar{\psi} \rightarrow \bar{\psi} e^{-i e(\Lambda+b S)}\right), \quad A_{\mu} \rightarrow A_{\mu}+\partial_{\mu} \Lambda, \\
A_{-} \rightarrow A_{-}+b q, \quad S \rightarrow S+C \text { for } \square^{2} \Lambda(x)=0\left(=\partial_{\mu} b=\partial_{\mu} C\right) .
\end{gathered}
$$

The above gauge freedom allows us to introduce a more general conformal transformation law for the basic fields which still respects the equations of motion. We shall use, in particular, a pure imaginary anomalous dimension for the spinor field (cf. [F7] and [B3(b)]):

$$
\begin{aligned}
& U(\varrho) \psi(x) U(\varrho)^{-1}=\varrho^{\frac{3}{2}+i e \delta} \psi(\varrho x), \\
& U(\varrho) \bar{\psi}(x) U(\varrho)^{-1}=\varrho^{\frac{3}{2}-i e \delta} \bar{\psi}(\varrho x) .
\end{aligned}
$$


It leads to a special conformal transformation law (for $h_{c}: x^{\mu} \rightarrow^{\prime} x^{\mu}=\omega\left(x^{\mu}+c^{\mu} x^{2}\right)$, where $\omega^{-1}=1+2 x c+c^{2} x^{2}$ ) that differs from the standard one, $\psi \rightarrow^{\prime} \psi$, by a gauge transformation:

$$
U\left(h_{c}\right) \psi U\left(h_{c}\right)^{-1}=\omega^{i e \delta \prime} \psi=\exp \left\{-i e \delta \ln \left(1+2 c x+c^{2} x^{2}\right)\right\}^{\prime} \psi ;
$$

it should be accompanied by an additive gauge transformation of the "compensating" (transformed) 4-potential ' $A_{\mu}$ :

$$
\begin{aligned}
U\left(h_{c}\right) A_{\mu} U\left(h_{c}\right)^{-1} & =A_{\mu}+\delta \partial_{\mu} \ln \omega \\
& =A_{\mu}-2 \delta \frac{c_{\mu}+c^{2} x_{\mu}}{1+2 c x+c^{2} x^{2}} .
\end{aligned}
$$

Note that $\Lambda(x)=\delta \ln \omega$ satisfies the restriction $\square^{2} \Lambda=0$ of $(2.23)$ for $\omega^{-1}(x, c)>0$.

We shall now demonstrate in a formal way (within the framework of the Feynman path integral - see, e.g., [P4]) that the non-conventional $\left(A_{-}-\right.$and $S$ dependent) terms in the Lagrangian (2.19) play the role of a gauge fixing.

We start with a modified form of the corresponding part of the action

$$
\begin{aligned}
I\left(A_{\mu}, J^{\nu} ; A_{-}, S ; a\right) \\
=\frac{\alpha}{2} a^{2}+\int\left\{\frac{\beta}{8}\left(A_{-}-a\right) \square^{2}\left(A_{-}-a\right)-\frac{1}{2}\left(A_{-}-a\right) \square \partial A\right. \\
\left.\quad+\frac{v}{2} S \square^{2} S-\left(A_{-}-a\right) \partial_{\mu} S J^{\mu}\right\} d^{4} x,
\end{aligned}
$$

where " $a$ " is a constant dynamical variable ("the value of $A_{-}$at infinity"). We define the generating functional

$$
\begin{aligned}
F_{h, H}\left(A_{\mu}, J^{v}\right)=N \int D S \int D\left(A_{-}-a\right) \int_{-\infty}^{\infty} d a \\
\quad \exp \left\{i\left(I\left(A_{\mu}, \ldots, a\right)+\int\left(h(x) A_{-}(x)+H(x) S(x)\right) d^{4} x\right)\right\} \\
=N_{1} \int D S \exp \left\{i \int \left(\frac{v}{2} S \square^{2} S+H S\right.\right. \\
\left.\left.\quad-\frac{2}{\beta}\left(\frac{1}{2} \square \partial A-J_{+}-h\right) \frac{1}{\square^{2}}\left(\frac{1}{2} \square \partial A-J_{+}-h\right)\right) d^{4} x-i \frac{\tilde{h}^{2}}{2 \alpha}\right\}, \\
\tilde{h}=\int h(x) d^{4} x,
\end{aligned}
$$

where $h(x)$ and $H(x)$ are external sources, $J_{+}=-J^{\mu} \partial_{\mu} S$ [see (2.18b)], $N$ and $N_{1}$ are (infinite!) normalization constants; $\frac{1}{\square^{2}}$ is the integral operator defined by

$$
\left(\frac{1}{\square^{2}} f\right)(x)=\frac{i}{(4 \pi)^{2}} \int \ln \frac{l^{2}}{(x-y)^{2}+i 0} f(y) d^{4} y .
$$

[It is independent of $l$ on the subspace $\mathscr{V}_{0}$ of functions $f$ with vanishing integral; it appears reasonable to assume that the external source $H(x)$ belongs to $\mathscr{V}_{0}$.] 
The average of (2.27) over the (local) gauge group gives

$$
\begin{aligned}
& \int F_{h, H}\left(A_{\mu}+\partial_{\mu} \Lambda, J^{v}\right) D \Lambda \\
& \quad=N_{2} e^{-i \frac{\tilde{h}^{2}}{2 \alpha} \int D S e^{i \int\left(\frac{v}{2} S \square^{2} S+H S\right) d^{4} x}} \\
& \quad=C \exp \left\{-\frac{i}{2}\left(\frac{\tilde{h}^{2}}{\alpha}+\frac{1}{v} \int H\left(\frac{1}{\square^{2}} H\right) d^{4} x\right)\right\},
\end{aligned}
$$

where $C$ is a constant. We can choose the normalization constant $N$ (respectively, $N_{1}$ and $N_{2}$ ) in such a way that $C=1$. We see, in particular, that for vanishing sources

$$
\int F_{0,0}\left(A_{\mu}+\partial_{\mu} \Lambda, J^{v}\right) D \Lambda=C(=1),
$$

which is (according to [P4]) precisely the condition that the contribution (2.26) to the action is a gauge fixing term. Moreover, it follows from (2.29) that the expectation value of a time-ordered product of gauge invariant fields (say, $F_{\mu \nu}$ and $J_{\lambda}$ ) and a number of fields $A_{-}$and $S$ factorizes into a product of the standard gauge invariant expectation values (of $T$-products of $F_{\mu \nu}$ 's and $J_{\lambda}$ 's) with generalized free type $\tau$-functions of $A_{-}$and $S$ that can be evaluated from (2.29). We have

$$
\begin{gathered}
<T J^{\lambda_{1}}\left(x_{1}\right) \ldots J^{\lambda_{m}}\left(x_{m}\right) F_{\mu_{1} v_{1}}\left(x_{m+1}\right) \ldots F_{\mu_{n} v_{n}}\left(x_{m+n}\right) e^{i \int(h(x) A-(x)+H(x) S(x)) d^{4} x} \\
=\left\langle T J^{\lambda_{1}}\left(x_{1}\right) \ldots F_{\mu_{n} v_{n}}\left(x_{m+n}\right)\right\rangle_{0} \\
\cdot \exp \left\{\frac{-i \widetilde{h}^{2}}{2 \alpha}+\frac{1}{2 v(4 \pi)^{2}} \iint H(x) \ln \frac{l^{2}}{(x-y)^{2}+i 0} H(y) d^{4} x d^{4} y\right\} \\
\left\langle T A_{-}(x) A_{-}(y)\right\rangle_{0}=\frac{i}{\alpha} \\
\langle T S(x) S(y)\rangle_{0}=\frac{1}{(4 \pi)^{2} v} \ln \frac{(x-y)^{2}+i 0}{l^{2}}
\end{gathered}
$$

We see that the $\tau$-functions of $A_{\text {- }}$ are only real for pure imaginary $\alpha$ 's [i.e. for a complex action (2.26)]. As we shall see [in Eq. (3.16) below] the 2-point function (2.32a) that is related by conformal invariance to the normalized transverse photon propagator corresponds to

$$
\alpha=-8 \pi^{2} i
$$

We shall take the above heuristic argument as a justification for postulating (2.31), (2.32).

\section{Quantization}

\section{A) General Requirements. Ward Identities. Electron Propagator}

The construction of a quantum field theory corresponding to a given set of classical field equations requires new postulates that include a choice of certain operator properties of the basic quantized fields (commutation relations, prop- 
erties of vacuum expectation values), and a proper definition of the (renormalized) composite fields (products of local field operators) which appear in the non-linear terms of the field equations. Looking for a conformal QED, we replace the canonical commutation relations of the standard (perturbative) approach by a number of requirements the most important of which is the conformal invariance of renormalized Green functions. This basic requirement implies that we are looking for a renormalization group fixed point (at least in the case when elementary representations of the subgroup of dilations are involved, leading to power laws for vertex functions) - see, e.g. [S2, M3, A1,2].

The conformal quantum postulates involve some freedom related to the choice of representations for renormalized Green functions. We shall start in this section with those basic properties, which, in our view, should take place in any conformal QED [related to the classical Lagrangian (2.19)] leaving the more debatable requirements to Sect. 3B. The postulates within each section are also arranged in decreasing generality.

We first assume that the classical field equations remain valid at the quantum level provided that the encountered composite operators are renormalized by using operator product expansions. We shall not attempt to formalize this requirement in general; its precise meaning in the context of the present model of massless QED will be made clear in Sect.4. We shall write the equations for Euclidean (Schwinger) functions which inherit the properties of time-ordered $(\tau$-)functions. They include differentiation of the $\theta$-function and use the equal time commutation relations for fields (like $J^{\mu}$ and $A_{-}$) whose dimensions remain canonical in the interacting case. Therefore, we find it convenient to set our basic postulates for (Minkowski space) time-ordered products of local field operators.

Rather than devising clumsy general formulations we shall write down an implication of the Maxwell equation (2.7), (2.15) for the time-ordered product of two gauge invariant fields.

Example 3.1. If $F_{\mu \nu}$ and $\partial_{\mu} A_{-}$transform under the non-decomposable 10dimensional representation of $\mathrm{SO}(4,2)$, described in Sect. 2, then we demand the validity of the following quantized form of the Maxwell equation:

$$
\begin{aligned}
& \frac{\partial}{\partial x_{\varrho}} T\left(F_{\lambda \varrho}(x) F_{\mu v}(y)\right)-\frac{1}{2} \square_{x} T\left(\partial_{\lambda} A_{-}(x) F_{\mu v}(y)\right) \\
& \quad=\left(\eta_{\lambda \mu} \partial_{v}-\eta_{\lambda v} \partial_{\mu}\right) \delta(x-y)+T\left(J_{\lambda}(x) F_{\mu v}(y)\right) .
\end{aligned}
$$

The $\delta$-function term is a covariant reflection of the equal-time commutation relations between $F_{\lambda 0}$ and $F_{\mu \nu}$.

Similarly, Eq. (2.15) applied to the Euclidean photon propagator should read

$$
\mathscr{M}(\partial)^{M}{ }_{N^{\prime}}\left\langle A^{N^{\prime}}(x) A_{N}(0)\right\rangle_{E}=\delta^{M}{ }_{N} \delta(x)+\left\langle J^{M}(x) A_{N}(0)\right\rangle_{E} .
$$

We shall assume throughout that we are working with the covariant $\left(T^{*}\right.$ - or Wick-) time-ordered product. The extension of a relation like (3.1) to products of several fields uses the standard algebraic properties of $T$-products. These include anticommutativity for any pair of local Fermi fields and commutativity of a local Bose field with any other local field as well as an associativity property which we 
shall write down in a special case to appear in our analysis of the Ward identity (WI) below:

$$
\begin{aligned}
T\left(\Psi\left(x_{1}\right) T\left(\bar{\psi}\left(x_{2}\right) J^{\mu}\left(x_{3}\right)\right)\right) & =T\left(T\left(J^{\mu}\left(x_{3}\right) \psi\left(x_{1}\right)\right) \bar{\psi}\left(x_{2}\right)\right) \\
& =T\left(\psi\left(x_{1}\right) \bar{\psi}\left(x_{2}\right) J^{\mu}\left(x_{3}\right)\right) .
\end{aligned}
$$

A central postulate of local QED is the operator WI

$$
\begin{gathered}
\frac{\partial}{\partial y^{\mu}}\left(T \psi(x) J^{\mu}(y)\right)=\delta\left(x^{0}-y^{0}\right)\left[J^{0}(y), \psi(x)\right]=-e \delta(x-y) \psi(x), \\
\frac{\partial}{\partial y^{\mu}}\left(T \bar{\psi}(x) J^{\mu}(y)\right)=\delta\left(x^{0}-y^{0}\right)\left[J^{0}(y), \bar{\psi}(x)\right]=e \delta(x-y) \bar{\psi}(x) .
\end{gathered}
$$

The equal-time current-field commutation, incorporated in (3.3), is the only trace of the canonical anticommutation relations $\left[\psi(t, \mathbf{x}), \psi^{*}(t, \mathbf{y})\right]_{+}=\delta(\mathbf{x}-\mathbf{y})$ (for $J^{0}(x)=i e: \bar{\psi}(x) \psi(x):=e: \psi^{*}(x) \psi(x):$ ). (These anticommutation relations will not be assumed for interacting fields, which will be allowed to acquire an anomalous dimension, while the property (3.3), characteristic for a current-charge density, will be retained.)

As a consequence of (3.3) we have the following standard WI relating the Euclidean 3- and 2-point (Schwinger) functions

$$
\frac{\partial}{\partial x_{3}^{\mu}}\left\langle\psi\left(x_{1}\right) \bar{\psi}\left(x_{2}\right) J^{\mu}\left(x_{3}\right)\right\rangle_{E}=i e S_{\psi}\left(x_{12}\right)\left[\delta\left(x_{23}\right)-\delta\left(x_{13}\right)\right]
$$

where

$$
S_{\psi}\left(x_{12}\right) \equiv\left\langle\psi\left(x_{1}\right) \bar{\psi}\left(x_{2}\right)\right\rangle_{E}, \quad x_{12} \equiv x_{1}-x_{2} .
$$

[The difference in the $i$-factor between (3.3) and (3.4) comes from the relation $\left(d_{4} p\right)_{M} \leftrightarrow i\left(d_{4} p\right)_{E}$ between Minkowski and Euclidean momentum space volume elements for $p^{0} \leftrightarrow i p_{4}$.]

Proposition 3.2. The field equations (2.15) and the WI (3.3) together with the assumption that $A_{-}(x)$ behaves like a constant at infinity and that $\psi(x)$ has an appropriate asymptotic behaviour, consistent with its conformal properties, imply the relations

$$
\begin{aligned}
& \left\langle\psi\left(x_{1}\right) \ldots \psi\left(x_{n}\right) \bar{\psi}\left(y_{1}\right) \ldots \bar{\psi}\left(y_{n}\right) A_{-}(z)\right\rangle_{E} \\
& \quad=\frac{e}{8 \pi^{2}}\left\langle\psi\left(x_{1}\right) \ldots \psi\left(x_{n}\right) \bar{\psi}\left(y_{1}\right) \ldots \bar{\psi}\left(y_{n}\right)\right\rangle_{E}\left(i \ln \frac{\left(y_{1}-z\right)^{2} \ldots\left(y_{n}-z\right)^{2}}{\left(x_{1}-z\right)^{2} \ldots\left(x_{n}-z\right)^{2}}+C_{n}\right) .
\end{aligned}
$$

Similarly,

$$
\begin{aligned}
& \left\langle\psi\left(x_{1}\right) \bar{\psi}\left(x_{2}\right) A_{-}\left(x_{3}\right) A_{-}\left(x_{4}\right)\right\rangle_{E} \\
& \quad=S_{\psi}\left(x_{12}\right)\left\{\left(\frac{e}{8 \pi^{2}}\right)^{2}\left(i \ln \frac{x_{23}^{2}}{x_{13}^{2}}+C\right)\left(i \ln \frac{x_{24}^{2}}{x_{14}^{2}}+C\right)+\left\langle A_{-}\left(x_{3}\right) A_{-}\left(x_{4}\right)\right\rangle_{E}\right\} .
\end{aligned}
$$

Proof. The divergence of $F^{\mu \nu}$ in the conformal Maxwell equation

$$
\partial_{v} F^{\mu \nu}-\frac{1}{2} \square \partial^{\mu} A_{-}=J^{\mu}
$$


does not contribute to the local charge density (see [F2, S5]). It then follows from (3.3) and (3.8) that

$$
\begin{gathered}
\frac{1}{2} \square_{y}^{2} T\left(\psi(x) A_{-}(y)\right)=i e \delta(x-y) \psi(x), \\
\frac{1}{2} \square_{y}^{2} T\left(\bar{\psi}(x) A_{-}(y)\right)=-i e \delta(x-y) \bar{\psi}(x) .
\end{gathered}
$$

Equations (3.6), (3.7) are an (Euclidean) integrated version of (3.9) that takes into account the asymptotic conditions and the identity $\square^{2} \ln \frac{l^{2}}{x^{2}}=(4 \pi)^{2} \delta(x)$ (where
is the Euclidean Laplacian).

Remark 3.3. Equation (3.9) corresponds to the following Minkowski space commutation relations:

$$
\begin{gathered}
{\left[A_{-}(x), \psi(y)\right]=\frac{e \varepsilon\left(x^{0}-y^{0}\right)}{2 \pi} \theta\left(-(x-y)^{2}\right) \psi(y),} \\
{\left[A_{-}(x), \bar{\psi}(y)\right]=-\frac{e \varepsilon\left(x^{0}-y^{0}\right)}{2 \pi} \theta\left(-(x-y)^{2}\right) \bar{\psi}(y),}
\end{gathered}
$$

where the coefficient in the right-hand side is related to the Pauli-Jordan commutator function $\mathscr{D}(x)$ by

$$
\square \frac{-\varepsilon\left(x^{0}\right)}{4 \pi} \theta\left(-x^{2}\right)=\mathscr{D}(x)=\frac{\varepsilon\left(x^{0}\right)}{2 \pi} \delta\left(x^{2}\right)=2 \pi i \int \varepsilon\left(p^{0}\right) \delta\left(p^{2}\right) e^{i p x} d_{4} p .
$$

We shall first use in an essential way the conformal invariance in determining the Dirac propagator.

C1. The 2n-point Schwinger functions $\left\langle\psi\left(x_{1}\right) \ldots \psi\left(x_{n}\right) \bar{\psi}\left(y_{1}\right) \ldots \bar{\psi}\left(y_{n}\right)\right\rangle_{E}$ are $\gamma_{5}-$ invariant and invariant with respect to the tensor product of $(2 n)$ elementary Diractype representation of the Euclidean conformal group $\operatorname{Spin}(5,1)$ corresponding to a (real) dimension $d$.

The general form of the conformal electron propagator (3.5) is given by (1.3). Remark 3.4. The 2-point Wightman function

$$
w_{\psi \psi^{*}}(x)=\frac{2 \pi C_{\psi}}{\Gamma\left(\frac{5}{2}-d\right) \Gamma\left(d-\frac{3}{2}\right)} \int\left(-p^{2}\right)_{+}^{d-5 / 2} \theta\left(p^{0}\right)\left(p^{0}-\gamma^{0} \gamma \cdot \mathbf{p}\right) e^{i p x} d_{4} p
$$

related to (1.3) is positive definite for $d \geqq \frac{3}{2}, C_{\psi}>0$. The vanishing of $w_{\psi \psi^{*}}$ for $d-\frac{5}{2}=0,1, \ldots$ is related to the assumption that $S_{\psi}$ is dilation invariant at these points. The anomalous dimension $d-\frac{3}{2}$ of the charged field is gauge dependent. A standard renormalization group analysis using second order calculations gives

$$
d-\frac{3}{2} \approx\left(\beta-\frac{1}{8 \pi^{2} v}\right) \frac{e^{2}}{(4 \pi)^{2}} .
$$

(For a similar computation in the framework of conventional massless QED see [A2, E1, F6].) We shall not exploit here the WI's associated with the conserved axial current. 


\section{B) Conformal QED with a Free Photon Propagator}

Proposition 3.5. The general conformal invariant 2-point function $\left\langle A^{M}(x) A_{N}(0)\right\rangle_{E}$ for the Spin(5,1)-representation, characterized by the infinitesimal law (2.4), is a multiple of the free photon Schwinger function

$$
\Delta_{A}(x)=\left\langle\left(\begin{array}{c}
A^{\mu}(x) \\
A_{-}(x)
\end{array}\right)\left(A_{v}(0) A_{-}(0)\right)\right\rangle_{E}=\int e^{i p x} \tilde{\Delta}_{A}(p) d_{4} p,
$$

where

$$
\tilde{\Delta}_{A}(p)=\left(\begin{array}{cc}
\frac{1}{p^{2}}\left(\delta_{v}^{\mu}-(1-\beta) \frac{p^{\mu} p_{v}}{p^{2}}\right) & 2 i \frac{p^{\mu}}{p^{4}} \\
-2 i \frac{p_{v}}{p^{4}} & -2 \pi^{2} \delta(p)
\end{array}\right)
$$

satisfies (for $\mathscr{M}$ defined in (2.14))

$$
\mathscr{M}(i p) \tilde{\Delta}_{A}(p)=\mathbb{1} \text {, so that } \mathscr{M}(\partial) \Delta_{A}(x)=\delta(x) .
$$

A proof of this statement based on the manifestly covariant formalism is given in Appendix B to [F7].

Remark 3.6. Equation (3.15) does not determine the coefficient to $\delta(p)$ in the righthand side of (3.14b). One way to verify that our choice $\left(-2 \pi^{2}\right)$ fits the requirement of conformal invariance is to exploit the infinitesimal law (2.4) and the condition $C_{\mu} \mid 0>=0$. The result is

$$
\left\langle A_{-}(x) A_{-}(0)\right\rangle_{0}=-\frac{1}{8 \pi^{2}}=\left\langle A_{-}(x) A_{-}(0)\right\rangle_{E}
$$

in accord with (3.14).

Corollary 3.7. Equation (3.14) together with the Euclidean Maxwell equations (3.1b) lead to trivial current-field and current-current 2-point functions:

$$
\left\langle J^{M}(x) A_{N}(0)\right\rangle_{E}=\mathscr{M}(\partial)^{M}{ }_{M^{\prime}} \Delta_{A}(x)^{M^{\prime}}{ }_{N}-\delta^{M}{ }_{N} \delta(x)=0
$$

(where $J^{-} \equiv J_{+}$) and hence

$$
\left\langle J^{M}(x) J_{N}(0)\right\rangle_{E}=0 .
$$

Remark 3.8. The current-current Wightman function evaluated in second order perturbation theory (using the free electron propagator (1.3) for $d=\frac{3}{2}$ ) is non-trivial and consistent with conformal invariance:

$$
\begin{gathered}
\left\langle J^{\mu}(x) J_{v}(0)\right\rangle_{0}=\frac{e^{2}}{\pi^{4}} \frac{r^{\mu}{ }_{v}(x)}{\left(x^{2}+i 0 x^{0}\right)^{3}}=\frac{e^{2}}{12 \pi^{4}}\left(\delta_{v}^{\mu} \square-\partial^{\mu} \partial_{v}\right) \frac{1}{\left(x^{2}+i 0 x^{0}\right)^{2}} \\
=\frac{e^{2}}{6 \pi} \int \theta_{+}(p)\left(p^{\mu} p_{v}-p^{2} \delta_{v}^{\mu}\right) e^{i p x} d_{4} p, \quad\left(\theta_{+}(p)=\theta\left(p^{0}\right) \theta\left(-p^{2}\right)\right) \\
r^{\mu}{ }_{v}=\delta^{\mu}{ }_{v}-2 \frac{x^{\mu} x_{v}}{x^{2}}
\end{gathered}
$$


However, its Euclidean counterpart is only covariant under a non-decomposable (logarithmic) representation of the dilation subgroup. Indeed, defining the finite part of $\frac{1}{x^{4}}$ by

$$
\left(\frac{1}{x^{4}}\right)_{\ell}=\lim _{\varepsilon \rightarrow+0} \frac{d}{d \varepsilon}\left(\frac{\varepsilon}{x^{4}}\left(\frac{x^{2}}{e^{2}}\right)^{\varepsilon}\right)
$$

we find

$$
\begin{aligned}
& \left\langle J^{\mu}(x) J_{v}(0)\right\rangle_{E}=\frac{e^{2}}{12 \pi^{2}}\left(\delta_{v}^{\mu} \square-\partial^{\mu} \partial_{v}\right)\left(\frac{1}{x^{4}}\right)_{\ell} \\
& =\frac{e^{2}}{12 \pi^{2}} \lim _{\varepsilon \rightarrow 0} \frac{d}{d \varepsilon}\left\{\frac{\Gamma(1+\varepsilon)}{\Gamma(2+\varepsilon)} \int \frac{p^{\mu} p_{v}-p^{2} \delta_{v}^{\mu}}{\left(4 \ell^{2} p^{2}\right)^{\varepsilon}} e^{i p x} d_{4} p\right\} \\
& =\frac{e^{2}}{12 \pi^{2}} \int\left(\ln \frac{\mu^{2}}{p^{2}}\right)\left(p^{\mu} p_{v}-p^{2} \delta_{v}^{\mu}\right) e^{i p x} d_{4} p
\end{aligned}
$$

(where the "subtraction point" $\mu$ is proportional to $\frac{1}{\ell}$ ). More troublesome, the invariant 2-point function $\left\langle J^{\mu}(x) J_{+}(y)\right\rangle_{0}$ is inconsistent with current conservation in that case. It is nevertheless clear that the vanishing of the 2-point functions (3.17), (3.18) is representation dependent.

Equation (3.18) was adopted as a unique possibility in the conformal QED of Johnson-Baker-Adler (JBA) (see [J1, A1,2, B1]). Although we leave open the possibility that (a modified) conformal invariance is still consistent with the more realistic ansatz (3.19), we shall only explore in this paper the simplest possibility (3.18).

C2. The photon propagator is invariant under the standard (non-decomposable) conformal law (2.3), (2.4).

As a consequence of Proposition 3.5 and of Corollary 3.6, this postulate leads to a JBA-type model of conformal QED.

\section{C3. The Schwinger functions}

$$
\left\langle\psi\left(x_{1}\right) \ldots \psi\left(x_{m}\right) \bar{\psi}\left(y_{1}\right) \ldots \bar{\psi}\left(y_{m}\right) J_{\left(z_{1}\right)}^{\mu_{1}} \ldots J_{\left(z_{n}\right)}^{\mu_{n}}\right\rangle_{E}
$$

are conformal and $\gamma_{5}$-invariant with respect to the tensor product of the standard elementary representation for $\psi$ (with dimension $d$ ) and $J^{\mu}$ (with dimension 3 ).

Corollary 3.9. It follows from (3.3), (3.10), and C3. that

$$
\begin{aligned}
& \left\langle\psi\left(x_{1}\right) \bar{\psi}\left(x_{2}\right) J^{\mu}\left(x_{3}\right)\right\rangle_{E} \\
& =i e C_{\psi} \frac{\Gamma\left(d+\frac{1}{2}\right)}{\Gamma\left(\frac{5}{2}-d\right)}\left(\frac{4}{x_{12}^{2}}\right)^{d-\frac{3}{2}}\left\{\frac{B+1}{2} S_{3 / 2}\left(x_{13}\right) \gamma^{\mu} S_{3 / 2}\left(x_{32}\right)\right. \\
& \left.\quad+(B-1) \frac{x_{12}}{x_{12}^{2}} \Delta\left(x_{13}\right) \overleftrightarrow{\partial_{3}^{\mu}} \Delta\left(x_{23}\right)\right\}, \quad\left(\Delta(x)=\frac{1}{4 \pi^{2} x^{2}}\right),
\end{aligned}
$$

where $B$ is an arbitrary (real) constant (see Eq. (IV.48) of [T1]). 
Remark 3.10. It should be noted that the 3-point function

$$
S_{-}\left(x_{1}, x_{2} ; x_{3}\right) \equiv\left\langle\psi\left(x_{1}\right) \bar{\psi}\left(x_{2}\right) A_{-}\left(x_{3}\right)\right\rangle_{E}=S_{\psi}\left(x_{12}\right)\left(\frac{i e}{8 \pi^{2}} \ln \frac{x_{23}^{2}}{x_{13}^{2}}+C\right)
$$

(obtained from (3.6) for $n=1$ ) is not invariant under the standard transformation law (2.22), (2.4) for $\psi$ and $A_{-}$. However, there exists a more general law which relates $S_{-}$and

$$
S_{v}\left(x_{1}, x_{2} ; x_{3}\right) \equiv\left\langle\psi\left(x_{1}\right) \bar{\psi}\left(x_{2}\right) A_{v}\left(x_{3}\right)\right\rangle_{E}
$$

with the longitudinal 3-point function

$$
l_{v}\left(x_{1}, x_{2} ; x_{3}\right) \equiv\left\langle\psi\left(x_{1}\right) \bar{\psi}\left(x_{2}\right) \partial_{v} S\left(x_{3}\right)\right\rangle_{E},
$$

and

$$
l_{-}\left(x_{1}, x_{2}\right) \equiv\left\langle\psi\left(x_{1}\right) \bar{\psi}\left(x_{2}\right) q\right\rangle_{E}=S_{\psi}\left(x_{12}\right) .
$$

(In the last equation we have used the ansatz $\psi \bar{\psi} q=\psi \bar{\psi}-$ cf. (2.18a).) A compact form of this general law is given in terms of the conformal inversion $I_{r} x=\frac{x}{x^{2}}$ (cf. $[T 1,2])$. The Euclidean picture fields would transform as follows under $I_{r}$ for elementary representations of the (extended) $\operatorname{Spin}(5,1)$ :

$$
\begin{gathered}
\psi(x) \rightarrow \psi^{(r)}(x)=i \gamma_{5} \frac{\not x}{\left(x^{2}\right)^{d+1 / 2}} \psi\left(I_{r} x\right), \\
\bar{\psi}(x) \rightarrow \bar{\psi}^{(r)}(x)=\bar{\psi}\left(I_{r} x\right) i \gamma_{5} \frac{x}{\left(x^{2}\right)^{d+1 / 2}}, \\
A_{-}(x) \rightarrow A_{-}^{(r)}(x)=A_{-}\left(I_{r} x\right), \quad A_{\mu} \rightarrow A_{\mu}^{(r)}(x)=\frac{r_{\mu \nu}(x)}{x^{2}} A^{v}\left(I_{r} x\right) \quad \text { etc. },
\end{gathered}
$$

where $r_{v}^{\mu}(x)$ is given by (3.20). Only $l_{-}\left(x_{1}, x_{2}\right)$ is invariant under this elementary law:

$$
l_{-}^{(r)}\left(x_{1}, x_{2}\right) \equiv\left\langle\psi^{(r)}\left(x_{1}\right) \bar{\psi}^{(r)}\left(x_{2}\right) q\right\rangle_{E}=l_{-}\left(x_{1}, x_{2}\right)\left(=S_{\psi}\left(x_{12}\right)\right) .
$$

The non-decomposable representation spanned by $\partial_{v} S$ and $q$ implies the following non-elementary covariance property for $l_{v}$ :

$$
l_{v}^{(r)}\left(x_{1}, x_{2} ; x_{3}\right)+\frac{2 x_{3 v}}{x_{3}^{2}} l_{-}\left(x_{1}, x_{2}\right)=l_{v}\left(x_{1}, x_{2} ; x_{3}\right) .
$$

It is straightforward to verify that the pair of functions $S_{-}$(3.23a) and $l_{-}$(3.23c) satisfy the $I_{r}$-covariance law,

$$
S_{-}^{(r)}\left(x_{1}, x_{2} ; x_{3}\right)-\frac{i e}{8 \pi^{2}} l_{-}\left(x_{1}, x_{2}\right) \ln \frac{x_{1}^{2}}{x_{2}^{2}}=S_{-}\left(x_{1}, x_{2} ; x_{3}\right) .
$$

The law for $S_{v}$ (proposed in [F7]) combines together the last two formulas:

$$
\begin{aligned}
& S_{v}^{(r)}\left(x_{1}, x_{2} ; x_{3}\right)-\frac{i e}{8 \pi^{2}} l_{v}\left(x_{1}, x_{2} ; x_{3}\right) \ln \frac{x_{1}^{2}}{x_{2}^{2}}+\frac{2 x_{3} v}{x_{3}^{2}} S_{-}\left(x_{1}, x_{2} ; x_{3}\right) \\
& \quad=S_{v}\left(x_{1}, x_{2} ; x_{3}\right) .
\end{aligned}
$$


C4. The Schwinger functions of $\psi, \bar{\psi} ; A_{v}, A_{-} ; \partial_{v} S, q$ are related among themselves via the conformal inversion according to Eqs. (3.25), (3.26) (and their extension to higher point Green functions).

There is a more complicated transformation law for the fields which makes all 3-point functions of the 5-potential invariant. For the dilation and the $I_{r}$-inversion of Euclidean picture spinor fields it reads [S3, F7]

$$
\begin{gathered}
U(\varrho) \psi(x) U(\varrho)^{-1}=\varrho^{3 / 2}: \varrho^{i e \hat{\delta}} \psi(\varrho x):, \\
U(\varrho) \bar{\psi}_{(x)} U(\varrho)^{-1}=\varrho^{3 / 2}: \varrho^{-i e \hat{\delta}} \bar{\psi}(\varrho x):, \\
U\left(I_{r}\right) \psi(x) U\left(I_{r}\right)^{-1}=i \gamma_{5} \frac{\not x}{x^{4}}:\left(\frac{1}{x^{2}}\right)^{i e \hat{\delta}} \psi\left(I_{r} x\right):
\end{gathered}
$$

while the 5-potential obeys the standard non-decomposable law,

$$
\begin{aligned}
& U\left(I_{r}\right) A_{-}(x) U\left(I_{r}\right)^{-1}=A_{-}\left(I_{r} x\right)\left(=A_{-}^{(r)}(x)\right), \\
& U\left(I_{r}\right) A_{v}(x) U\left(I_{r}\right)^{-1}=A_{v}^{(r)}(x)+\frac{2 x_{v}}{x^{2}} A_{-}(x) .
\end{aligned}
$$

Here the normal products are defined as usual:

$$
: \varrho^{i e \hat{\delta}} \psi(x):=\varrho^{i e \delta^{(+)}} \psi(x) \varrho^{i e \delta^{(-)}},
$$

where

$$
\delta^{(-)}|0>=0=<0| \delta^{(+)}, \quad \delta^{(-)}=\delta^{(+)^{*}} .
$$

Although local fields either strictly anticommute (for a pair of Fermi fields) or strictly commute (otherwise) under the sign of an Euclidean expectation value, positive and negative frequency parts, in general, do not commute with the fields. Thus, in order to apply the operator law (3.27) to the evaluation of Schwinger functions, we should define the commutation relations of $\delta^{( \pm)}$between each other and with the local fields.

We shall set

$$
\begin{gathered}
{\left[\delta^{(-)}, \delta^{(+)}\right]=0, \quad\left[\delta^{(\mp)}, \psi(x)\right]= \pm \frac{i e b}{(4 \pi)^{2}} \psi(x),} \\
{\left[\delta^{( \pm)}, \bar{\psi}(x)\right]= \pm \frac{i e b}{(4 \pi)^{2}} \psi(x),}
\end{gathered}
$$

so that

$$
\begin{gathered}
\varrho^{-i e \delta(+)} \psi(x) \varrho^{i e \delta(+)}=\varrho^{-\frac{e^{2} b}{(4 \pi)^{2}}} \psi(x) \quad \text { etc. }, \\
{\left[\delta^{(\mp)}, A_{-}(x)\right]= \pm \frac{1}{8 \pi^{2}} q, \quad\left[\delta^{(\mp)}, A_{\mu}(x)\right]= \pm \frac{1}{8 \pi^{2}} \partial_{\mu} S(x) .}
\end{gathered}
$$

The electron propagator (1.3) is now reproduced from (3.26-3.28) provided that

$$
d=\frac{3}{2}+\frac{e^{2}}{(4 \pi)^{2}} b
$$


Comparing with (3.13), we see that $b$ plays the role of an overall gauge parameter. Furthermore, if we require

$$
\psi(x) \bar{\psi}(y) q=q \psi(x) \bar{\psi}(y)=\psi(x) \bar{\psi}(y)
$$

(in accord with (2.18)), we rederive the expression (3.23a) for $S_{-}$(up to a normalization constant) from the invariance requirement under the above operator law. In order to reproduce also the covariance property $(3.25 \mathrm{~b})$, we demand that

$$
\left[\delta^{( \pm)}, \partial_{\mu} S(x)\right]=0=\left[\delta^{( \pm)}, q\right]
$$

Proposition 3.11. Under the above assumptions the general conformal invariant 3point Schwinger function of $\partial_{\mu} S$, consistent with (2.16) and (3.34), is

$$
\left\langle\psi\left(x_{1}\right) \bar{\psi}\left(x_{2}\right) \partial_{\mu} S\left(x_{3}\right)\right\rangle_{E}=S_{\psi}\left(x_{12}\right)\left\{-\left(\frac{x_{13} \mu}{x_{13}^{2}}+\frac{x_{23} \mu}{x_{23}^{2}}\right)+\alpha \lambda_{\mu}\right\} \text {. }
$$

Equations (3.32) and (3.36) imply

$$
\begin{aligned}
S_{\mu}\left(x_{1}, x_{2} ; x_{3}\right)= & \frac{i e}{8 \pi^{2}} S_{\psi}\left(x_{12}\right)\left\{\left(\alpha \lambda_{\mu}-\frac{x_{13} \mu}{x_{13}^{2}}-\frac{x_{23} \mu}{x_{23}^{2}}\right) \ln \frac{x_{23}^{2}}{x_{13}^{2}}+A \lambda_{\mu}\right\} \\
& -i C_{\psi} e \frac{B+1}{8 \pi^{2}}\left(d-\frac{1}{2}\right) \Delta_{d-1 / 2}\left(x_{12}\right) \frac{x_{13}}{x_{13}^{2}} \gamma_{\mu} \frac{x_{23}}{x_{23}^{2}},
\end{aligned}
$$

where the coefficient to the last term is chosen to agree with (3.22); here $\Delta_{\delta}$ is given by (1.2) and

$$
\lambda_{\mu}=\frac{x_{13} \mu}{x_{13}^{2}}-\frac{x_{23} \mu}{x_{23}^{2}}\left(=\frac{1}{2} \partial_{3 \mu} \ln \frac{x_{23}^{2}}{x_{13}^{2}}\right) .
$$

The proof of this proposition is straightforward.

\section{C) Subspace of Physical 0-Charge States}

Assumption C2 that leads to a free photon propagator allows an explicit construction of the physical subspace (of the indefinite metric space for the local gauge under consideration) at least in the zero-charge sector.

We define the submanifold $\mathscr{D}^{\prime}$ of physical states of the field domain $\mathscr{D}$ as a maximal conformally invariant linear subspace of vectors $\Phi, \Psi$ of $\mathscr{D}$ such that

$$
\left\langle\Phi\left|A_{-}(x)\right| \Psi\right\rangle=0=\left\langle\Phi\left|\partial_{v} S\right| \Psi\right\rangle=\langle\Phi|q| \Psi\rangle .
$$

Remark 3.12. Equation (3.39) excludes from $\mathscr{D}^{\prime}$ vectors of the form $A_{-} \mid 0>$ or $\partial_{v} S \mid 0>$ that would have violated the non-negativity of the inner product. The positivity violation for $A_{-} \mid 0>$ is manifest from (3.16); for $\partial_{v} S$ it is derived from the expression for the 2-point Wightman function (cf. (2.32))

$$
\begin{aligned}
\left\langle\partial_{\mu} S(x) \partial_{\nu} S(0)\right\rangle_{0} & =\frac{r_{\mu \nu}(x)}{8 \pi^{2} v\left(x^{2}+i 0 x^{0}\right)} \\
& =\frac{2 \pi}{v} \int \theta\left(p^{0}\right) p_{\mu} p_{\nu} \delta^{\prime}\left(-p^{2}\right) e^{i p x} d_{4} p .
\end{aligned}
$$


Let $\mathscr{D}_{0}^{\prime}$ be the part of the 0 -charge sector of $\mathscr{D}^{\prime}$, generated by the action to the vacuum of polynomials of local fields smeared by test functions with compact support.

Proposition 3.13. $\mathscr{D}_{0}^{\prime}$ is generated by polynomials of $F_{\mu v}, \partial_{v} A_{-}$and $\square^{2} S$, the states $\partial_{v} A_{-}\left|0>, \partial_{v} F^{\mu v}\right| 0>$ and $\square^{2} S \mid 0>$ having zero norm.

The proposition follows from the factorization postulate (2.31) and from the properties of (free) 2-point functions of the encountered (Bose) fields. (We note that Eq. (3.39) does not contradict our assumption (2.18a) because of the vanishing of the current matrix elements in $\mathscr{D}^{\prime}$, which follows from (3.18) - see [S4].)

\section{Renormalized Schwinger-Dyson Equations (S-D Eqs)}

\section{A) Equations Involving 2- and 3-Point Functions}

We follow here the standard scheme of defining the renormalized S-D Eqs - see [S6, B5] (as well as [Z2]).

Let $F$ be an arbitrary polynomial in the fields $A_{N}, \partial_{\mu} S, \psi, \bar{\psi}$ and their derivatives: Eqs. (2.15), (2.18b), (2.20), (2.21) imply the following set of renormalized S-DEqs for Schwinger functions (since we only deal with Euclidean Green functions in this section, we skip the subscript $E$ in the expectation values):

$$
\begin{gathered}
\not\langle\psi(x) F\rangle-\left\langle\frac{\partial F}{\delta \bar{\psi}}\right\rangle=i e\langle N(h(x) \psi(x)) F\rangle, \\
h_{\mu}(x)=A_{\mu}(x)-A_{-}(x) \partial_{\mu} S(x), \\
\left(\partial^{\mu} \partial_{v}-\square \delta_{v}^{\mu}\right)\left\langle A^{v}(x) F\right\rangle-\frac{1}{2} \partial^{\mu} \square\left\langle A_{-}(x) F\right\rangle-\left\langle\frac{\delta F}{\delta A_{\mu}(x)}\right\rangle=\left\langle J^{\mu}(x) F\right\rangle,(4) \\
\frac{1}{2} \square \partial_{\mu}\left\langle A^{\mu}(x) F\right\rangle-\frac{\beta}{4} \square^{2}\left\langle A_{-}(x) F\right\rangle-\left\langle\frac{\delta F}{\delta A_{-}(x)}\right\rangle=-\left\langle N\left(\partial^{\mu} S(x) J_{\mu}(x)\right) F\right\rangle, \\
v \square \square^{2}\langle S(x) F\rangle-\left\langle\frac{\delta F}{\delta S(x)}\right\rangle=\left\langle\partial_{\mu} N\left(A_{-}(x) J^{\mu}(x)\right) F\right\rangle
\end{gathered}
$$

where

$$
J_{\mu}(x)=i e N\left(\bar{\psi}(x) \gamma_{\mu} \psi(x)\right)
$$

The normal products $N(.$.$) will be determined in a non-perturbative way as finite$ parts of Wilson expansions inserted in conformal invariant Schwinger functions. The remaining freedom will be restricted by the requirement of gauge invariance.

Proceeding to the study of the Dirac equation (4.1a) we shall use (and extend) some of the techniques of [F4, P1] (devised for the study of the 2-dimensional Thirring model). We define the smeared composite field $\psi_{1}(x, \varepsilon)$ by

$$
\left\langle\psi_{1}(x, \varepsilon) F\right\rangle=i e Z_{2}(\varepsilon) \int d \Omega_{z}\langle h(x+\varepsilon z) \psi(x) F\rangle-\left(Z_{2}(\varepsilon)-1\right) \not \partial\langle\psi(x) F\rangle,
$$


where $d \Omega_{z}$ is the normalized measure on $S^{3}$ :

$$
\int d \Omega_{z} f(z)=\frac{1}{\pi^{2}} \int \delta\left(z^{2}-1\right) f(z) d^{4} z .
$$

The field $h_{\mu}=q A_{\mu}-A_{-} \partial_{\mu} S$ transforms "homogeneously" [i.e. under an elementary representation of $\operatorname{Spin}(5,1)]$. The factor $q$ can be dropped [as in (4.1a)] when multiplied by a pair of conjugate spinor fields [see (3.34)].

The renormalization constant $Z_{1}(\varepsilon)$ does not appear, since we have set $Z_{1}=Z_{2}$ as required by gauge invariance.

Setting $F=\bar{\psi}(y)$ we can rewrite Eq. (4.1a) in the form

$$
\not_{x}\langle\psi(x) \bar{\psi}(y)\rangle-\delta(x-y)=\lim _{\varepsilon \rightarrow 0}\left\langle\psi_{1}(x, \varepsilon) \bar{\psi}(y)\right\rangle .
$$

(The $\delta$-function, which fits the free field limit for $e \rightarrow 0$, is cancelled by a similar term in the right-hand side (RHS) in the case when $\Psi$ acquires anomalous dimension.) The 3-point function $\left\langle h_{\mu}(x+\varepsilon z) \psi(x) \bar{\psi}(y)\right\rangle$ [which appears in (4.3) if we insert $\psi_{1}$ from (4.2)] is obtained from (3.37) by deleting the logarithmic term and replacing $\frac{1+B}{2}$ and $A$ by two arbitrary constants $g_{1}$ and $g_{2}$. It is clear [taking into account the composite character of the second term in $\left.h_{\mu}(4.1 \mathrm{a})\right]$ that one should actually deal with the 4-point function

$$
S_{\mu}^{(4)}=\left\langle A_{-}\left(x_{1}\right) \partial_{\mu} S\left(x_{2}\right) \psi\left(x_{3}\right) \bar{\psi}\left(x_{4}\right)\right\rangle
$$

in the limit $x_{12} \rightarrow 0$.

The small $\varepsilon$ behaviour of the spherically smeared 3-point function ${ }^{2}$

$$
G(x, p ; \varepsilon)=\int d^{4} y \int d \Omega_{z}\langle h(x+\varepsilon z) \psi(x) \bar{\psi}(y)\rangle e^{-i p y}
$$

involved in (4.3) is analysed in the Appendix. The final expression (A.7) contains two terms of the type appearing in the left-hand side of (4.3), where we insert the conformal invariant 2-point function (1.3). In this way we obtain the following relations between the anomalous dimension $\lambda=d-\frac{3}{2}$, the $\psi$-field renormalization constant $Z_{2}$ and the free parameters $g_{1}$ and $g_{2}$ in the conformal invariant expression for $G$ :

$$
\begin{gathered}
\lambda\left(\equiv d-\frac{3}{2}\right)=\frac{e^{2}}{(4 \pi)^{2}}\left(g_{1}+g_{2}\right), \\
Z_{2}(\varepsilon, \lambda)=z(\lambda)\left(\frac{\varepsilon^{2}}{4}\right)^{\lambda} \quad\left(Z_{2}(\varepsilon, 0)=z(0)=1 \quad \text { for } \quad C_{\psi}(0)=1, e^{2}=0\right),
\end{gathered}
$$

where the product of $z(\lambda)$ with the normalization constant $C_{\psi}$ of the electron propagator $(1.3)$ is

$$
C_{\psi} z(\lambda)=\frac{1}{2+\left(2 g_{1}-g_{2}\right) \frac{e^{2}}{(4 \pi)^{2}}} \frac{\Gamma(3-\lambda)}{\Gamma(1+\lambda)} .
$$

2 Note that a similar analysis of the Dirac equation in the framework of [F3, P2] is hindered by the non-local character of the photon 3-point function 
Remark 4.1. The Euclidean counterpart of the more complicated action (2.26) leads to a somewhat modified system of equations. In particular, a term $\left\langle a \not \partial S\left(x_{1}\right) \psi\left(x_{1}\right) \bar{\psi}\left(x_{2}\right)\right\rangle$ is added to the RHS of the Dirac equation (4.3). The new system includes one more equation, obtained by varying with respect to $a$ :

$$
\left(8 \pi^{2}\right)\langle a F\rangle+\left\langle\frac{\delta F}{\delta a}\right\rangle+\int d^{4} x\left(\frac{1}{2} \square \partial_{\mu}\left\langle A^{\mu}(x) F\right\rangle-\left\langle J_{+}(x) F\right\rangle\right)=0 .
$$

(We have used that $\int \square^{2}\left\langle A_{-}(x) F\right\rangle d^{4} x=0$ according to (4.1a) and the Ward identities.) Combined with (4.1b) this equation leads to the relations

$$
\begin{gathered}
\langle a a\rangle=\left\langle a A_{-}\right\rangle=\left\langle A_{-} A_{-}\right\rangle\left(=-\frac{1}{8 \pi^{2}}\right), \\
\langle a F\rangle=0 \quad \text { if } \quad\left\langle\frac{\delta F}{\delta A_{-}}\right\rangle=0=\left\langle\frac{\delta F}{\delta a}\right\rangle .
\end{gathered}
$$

As a result, the Dirac equation (4.3) remains unchanged.

Turning next to the definition of the current, we set

$$
\begin{aligned}
& \left\langle J^{\mu}(x, \varepsilon) F\right\rangle \\
& \quad=i e Z_{2}(\varepsilon, \lambda) \int d \Omega_{z}\left\langle\bar{\psi}(x+\varepsilon z) \gamma^{\mu} \psi(x) F\right\rangle \\
& \quad-\left\langle\left\{\mathscr{D}_{v}^{\mu}(\varepsilon, \partial) A^{v}(x)+\mathscr{D}_{1}^{\mu}(\varepsilon, \partial) A_{-}(x)+\mathscr{D}_{2}^{\mu}(\varepsilon, \partial) S(x)\right\} F\right\rangle,
\end{aligned}
$$

where

$$
\begin{gathered}
\mathscr{D}_{v}^{\mu}=\frac{a_{1}}{\varepsilon^{2}} \delta_{v}^{\mu}+a_{2} \partial^{\mu} \partial_{v}+a_{3} \square \delta_{v}^{\mu}, \\
\mathscr{D}_{i}^{\mu}=\frac{b_{i}}{\varepsilon^{2}} \partial^{\mu}+C_{i} \square \partial^{\mu}, \quad i=1,2 .
\end{gathered}
$$

According to our assumption $\mathrm{C} 2$ about the conformal transformation properties of the photon propagator and Proposition 3.5, the charge renormalization constant $Z_{3}$ is finite and can, therefore, be set equal to 1 . We should like to give meaning to the current $J^{\mu}(x)$ in the Maxwell equation (4.1b) as a limit for $\varepsilon \rightarrow 0$ of (4.7a). Taking the divergence of (4.1b) for $F=F(y)=A_{v}, A_{-}$or $\partial_{v} S$ and using current conservation, we find the free field relation

$$
\frac{1}{2} \square_{x}^{2}\left\langle A_{-}(x) F(y)\right\rangle+\frac{\partial}{\partial x^{\mu}}\left\langle\frac{\delta F}{\delta A_{\mu}(x)}\right\rangle=0,
$$

which is satisfied by the conformal invariant 2-point functions (3.14). It follows that momentum space 2-point functions satisfy

$$
\begin{aligned}
& \int e^{-i p y}\left(\delta_{\sigma}^{\mu}-\frac{p^{\mu} p_{\sigma}}{p^{2}}\right)\left\{p^{2}\left\langle A^{\sigma}(x) F(y)\right\rangle-\frac{\delta F(y)}{\delta A_{\sigma}(x)}\right\} d^{4} y \\
& \quad=\lim _{\varepsilon \rightarrow 0} \int e^{-i p y}\left\langle J^{\mu}(x, \varepsilon) F(y)\right\rangle d^{4} y .
\end{aligned}
$$

The left-hand side of this equation vanishes identically for our choice of the photon propagator. Before studying the implications of this fact to the RHS we shall make a few comments. 
Remark 4.2. Finite subtractions appear, as discussed in [B5], in order to maintain the properties of the current functions dictated by gauge invariance which is destroyed by our regularization procedure. The type of the operators $\mathscr{D}_{i}$ is restricted by the asymptotic behaviour of the first term in (4.7a). More subtractions are possible in principle, e.g. cubic terms of the type $\mathscr{D}_{\mu \varrho \gamma \sigma} A^{\varrho}(x-\varepsilon z) A^{\gamma}(x) A^{\sigma}(x+\varepsilon z)$ [B5]. We neglect them since in our case they factorize in the average and do not give new structures.

Remark 4.3. The definition (4.7-4.8) of the current applies only if $F$ is a polynomial of basic fields. If $F$ includes composite operators, additional (contact) terms are needed in general (see, e.g., [Z2]). (This remark applies of course to all composite operators in the model.) We shall give only an example of functions involving two current operators:

$$
\begin{aligned}
\left\langle J_{\mu}\left(x_{1}\right) J_{v}\left(x_{2}\right) F\right\rangle & \\
= & \lim _{\varepsilon \rightarrow 0}\left\{\left\langle J_{\mu}\left(x_{1}, \varepsilon\right) J_{v}\left(x_{2}\right) F\right\rangle-\int d \Omega_{z}\left[D_{\mu \nu \gamma \varrho}^{1}\left(\varepsilon, x_{12}\right)\right.\right. \\
& \cdot\left\langle A^{\gamma}\left(x_{2}+\varepsilon z\right) A^{\varrho}\left(x_{2}\right) F\right\rangle+D_{\mu \nu \gamma}^{2}\left(\varepsilon, x_{12}\right)\left\langle A_{\left(x_{2}\right)}^{\gamma} F\right\rangle \\
& \left.\left.+D_{\mu \nu}^{3}\left(\varepsilon, x_{12}\right)\langle F\rangle\right]\right\} .
\end{aligned}
$$

In particular, for $F=\mathbb{1}$, we have

$$
D_{\mu \nu \gamma \varrho}^{1}=\gamma_{1} \eta_{\mu \gamma} \eta_{v \varrho} \delta\left(x_{2}\right) ; \quad D_{\mu \nu}^{3}=\left[\gamma_{2} \partial_{\mu} \partial_{v}+\gamma_{3}\left(\partial_{\mu} \partial_{\nu}-\square \eta_{\mu \nu}\right)\right] \delta\left(x_{12}\right) .
$$

The asymptotic behaviour of the 3-point functions involved in the RHS of (4.8) as well as the contribution of the subtraction terms are evaluated in the Appendix. We write here only the expression for the 3-point function of $A_{\mu}$ [see (3.37) with $\alpha=0]$ :

$$
\begin{aligned}
i e Z_{2}(\varepsilon, \lambda) \int d \Omega_{z} e^{-i p y}\left\langle\bar{\psi}(x+\varepsilon z) \gamma_{\mu} \psi(x) A_{\nu}(y)\right\rangle d^{4} y \\
=-\frac{e^{2} C_{\psi} z(\lambda)}{(2 \pi)^{2}} \frac{\Gamma(\lambda+2)}{\Gamma(1-\lambda)} e^{-i p x} \\
\quad \cdot\left\{\frac{2}{\varepsilon^{2} p^{2}} \eta_{\mu \nu}+2 \frac{(2 B+A)}{\varepsilon^{2}} \frac{p_{\mu} p_{v}}{p^{4}}+\left(\frac{1-A}{4}-\frac{1+B}{4}\right) \frac{p_{\mu} p_{v}}{p^{2}}\right. \\
\left.\quad+\alpha_{0} \eta_{\mu \nu}+\left(\frac{1+B}{8}+\frac{1}{12}\right) \eta_{\mu \nu} \ln \frac{p^{2} \varepsilon^{2}}{4}\right\}+0\left(\varepsilon^{2}\right) .
\end{aligned}
$$

Here $\alpha_{0}$ is a numerical constant [see (A.11)]. All terms in (4.10) except the last one are compensated by the contribution of the subtractions in (4.8) leading to some relations for the arbitrary constants in (4.7). These constants are completely determined when the other two equations in (4.8) (for $F=A_{-}$or $\partial_{\mu} S$ ) are also taken into account. There is no way, however, to cancel the finite transverse last term in (4.10) unless

$$
e^{2} z(\lambda) \frac{\Gamma(2+\lambda)}{\Gamma(1-\lambda)} C_{\psi}\left(\frac{1+B}{2}+\frac{1}{3}\right)=\frac{e^{2}}{2} \frac{\left(1-\lambda^{2}\right)(2-\lambda)}{2+\left(2 g_{1}-g_{2}\right) \frac{e^{2}}{(4 \pi)^{2}}}\left(B+\frac{5}{3}\right)=0,
$$

i.e. either $B=-\frac{5}{3}$ or $e\left(1-\lambda^{2}\right)(2-\lambda)=0$. 
Remark 4.4. Concerning the first possibility $\left(B=-\frac{5}{3}\right)$, we notice that it is not available in the framework of $[\mathrm{F} 3, \mathrm{P} 2, \mathrm{~K} 1]$; as compared to this framework we have a second independent transverse structure in the 3-point function (3.37). The above result can also be obtained from the Maxwell equation for $\left\langle A_{\mu} J_{v}\right\rangle$ [see (A.12)]. The finite contribution of the current 3-point function (3.22) to the Maxwell equation has been evaluated earlier in [M4] where a similar condition for $B$ has been put forward. [It is, however, incorrectly assumed in [M4] that the conformal invariant current Green's function $\left\langle T J_{\mu}(x) J_{\nu}(0)\right\rangle_{0}$ is proportional to the associate homogeneous distribution (3.21).] A number of the above formulas (as well as those of the Appendix) have been obtained in an inconclusive earlier attempt [D5] to tackle conformal $\mathrm{QED}^{3}$ ).

Regarding the current as an elementary conformal field we easily analyse also the last two equations (4.1c, d) for the various 2-point functions $\left\langle\partial_{\mu} S(x) F(y)\right\rangle$ and $\left\langle A_{-}(x) F(y)\right\rangle$ and prove (exploiting current conservation) their consistency with the (free) expressions (2.32), (3.14). The implications of the composite structure of $J^{\mu}(4.7)$ is further discussed in Sect. 4B.

\section{B) Equations Involving 4-Point Functions}

The Maxwell equations (4.1b) with $F \rightarrow F \psi \bar{\psi}$ and $F=A_{v}, \partial_{\mu} S$ or $A_{-}$together with the corresponding Ward identities imply the equations:

$$
\begin{aligned}
& \frac{1}{2} \square^{2}\left\langle A_{-}\left(x_{4}\right) F\left(x_{3}\right) \psi\left(x_{1}\right) \bar{\psi}\left(x_{2}\right)\right\rangle+\left\langle\frac{\delta F\left(x_{3}\right)}{\delta A_{\mu}\left(x_{4}\right)} \psi\left(x_{1}\right) \bar{\psi}\left(x_{2}\right)\right\rangle \\
& \quad=i e\left[\delta\left(x_{14}\right)-\delta\left(x_{24}\right)\right]\left\langle F\left(x_{3}\right) \psi\left(x_{1}\right) \bar{\psi}\left(x_{2}\right)\right\rangle .
\end{aligned}
$$

In agreement with Proposition 3.2 we have the following

Proposition 4.1. Let $F$ be either $A_{\mu}, \partial_{\mu} S$ or $A_{-}$. Then

$$
\begin{aligned}
\mathscr{S}_{F}= & \left\langle A_{-}\left(x_{4}\right) F\left(x_{3}\right) \psi\left(x_{1}\right) \bar{\psi}\left(x_{2}\right)\right\rangle \\
= & \left(C+\frac{i e}{8 \pi^{2}} \ln \frac{x_{42}^{2}}{x_{41}^{2}}\right)\left\langle F\left(x_{3}\right) \psi\left(x_{1}\right) \bar{\psi}\left(x_{2}\right)\right\rangle \\
& +\left\langle\psi\left(x_{1}\right) \bar{\psi}\left(x_{2}\right)\right\rangle\left\langle A_{-}\left(x_{4}\right) F\left(x_{3}\right)\right\rangle .
\end{aligned}
$$

The last term is present only for $F=A_{\mu}$ or $A_{-}$.

One can check that no finite part of $\mathscr{S}_{F}$ survives when $x_{2} \rightarrow x_{1}, x_{4} \rightarrow x_{1}$. Hence there is no contribution to the R.H.S. of Eq. (4.1d) for $F=A_{\mu}, \partial_{\mu} S$ or $A_{-}$. This shows that the composite character of the current does not affect the conclusion of the preceding section.

We let $x_{4} \rightarrow x_{3}$ in (4.13) for $F=\partial_{\mu} S$ thus getting

$$
\left\langle A_{-}\left(x_{3}\right) \partial_{\mu} S\left(x_{3}\right) \psi\left(x_{1}\right) \bar{\psi}\left(x_{2}\right)\right\rangle=\left(C+\frac{i e}{8 \pi^{2}} \ln \frac{x_{32}^{2}}{x_{31}^{2}}\right)\left\langle\partial_{\mu} S\left(x_{3}\right) \psi\left(x_{1}\right) \bar{\psi}\left(x_{2}\right)\right\rangle .
$$

If we choose $\alpha=0$ in the expression (3.36) for $\left\langle\partial_{\mu} S \psi \bar{\psi}\right\rangle$, (4.14) has the general form of the 3-point function (3.37) with $1+B=0=A$. Hence, as discussed in Sect. 4A, it

3 The model in [D5] reproduces the standard 3-point current function and an expression for $\left\langle A_{\mu} \psi \bar{\psi}\right\rangle$ which differs from (3.37) by purely longitudinal terms. However, the normal product in the Dirac equation requires an unconventional subtraction, which is avoided here 
just cancels down the contribution of the inhomogeneous term in (3.37) in the Dirac equation. Then we finally have in (4.4)

$$
g_{1}=\frac{1+B}{2}, \quad g_{2}=A \text {. }
$$

Combining (4.15) with (A.13), we obtain

$$
\lambda\left(=d-\frac{3}{2}\right)=\frac{e^{2}}{(4 \pi)^{2}}(\beta+\text { const }) .
$$

The fact that (4.14) does not contain the standard transverse part of (3.37) leads us to the following important conclusion.

Proposition 4.2. The Maxwell equation (4.1b) with $F=A_{-}\left(x_{1}\right) \partial_{\mu} S\left(x_{3}\right)$ (or $\left.F=\left(A_{\mu}-h_{\mu}\right)\left(x_{3}\right)\right)$ is not satisfied unless

$$
e C_{\psi} z(\lambda) \frac{\Gamma(\lambda+2)}{\Gamma(1-\lambda)}=\frac{e(2-\lambda)\left(1-\lambda^{2}\right)}{2+\left(2 g_{1}-g_{2}\right) \frac{e^{2}}{(4 \pi)^{2}}}=0
$$

The proof concerning the 3-point function (4.14) repeats the derivation of (4.10), (4.11).

Thus, the only way to cancel the $\log p^{2}$-term is to have either a trivial theory (with $e \psi=0$ - see the discussion in the Introduction) or a special gauge in which the anomalous dimension has the (negative) value $\lambda \rightarrow-1$.

\section{Appendix A. Small Distance Expansions of 3-Point Functions}

Our objective in this appendix is to evaluate the leading terms for $\varepsilon \rightarrow 0$ of smeared 3 -point functions of the type of $G(x, p ; \varepsilon)(4.4)$. Using the identity

$$
\frac{\not x}{x^{2}}=\lim _{\delta \rightarrow 0}\left(\frac{1}{2 \delta} \not \partial x^{2 \delta}\right)
$$

we can write the general conformal invariant expression of the integrand in (4.4) as the limit of

$$
\begin{aligned}
\left\langle h\left(x_{3}\right) \psi\left(x_{1}\right) \bar{\psi}\left(x_{2}\right)\right\rangle_{\delta} \\
=\frac{i e C_{\psi}}{(2 \pi)^{2}}\left\{-\frac{1}{2 \delta}\left(g_{1}(2 d-1) \frac{\not x_{13}}{x_{13}^{2}} \not_{3}+\frac{1}{2} g_{2} \not_{3} \not \phi_{1}\right) \Delta_{d-1 / 2}\left(x_{12}\right)\right. \\
\left.\cdot\left(\frac{x_{23}^{2}}{2}\right)^{\delta}+\frac{1}{2} g_{2} \frac{\not x_{13}}{x_{13}^{2}} S_{d}\left(x_{12}\right)\right\} \text { for } \delta \rightarrow 0 .
\end{aligned}
$$

Setting $x_{1}=x, x_{3}=x+\varepsilon z$, and taking the Fourier transform in $x_{2}=y$, we obtain

$$
\begin{aligned}
G(x, p ; \varepsilon)= & \frac{i e C_{\psi} e^{-i p x}}{(2 \pi)^{2} 2^{1-\lambda}} \frac{\Gamma(\lambda+1)}{\Gamma(1-\lambda)} \lim _{\delta \rightarrow 0} \frac{1}{\delta} \int d \Omega_{z}\left\{g_{1}(\lambda+1) \frac{\not}{\varepsilon^{2} z^{2}} \oiint_{z}\right. \\
& \left.+g_{2} \frac{1}{2 \varepsilon^{2}}\left(i \varepsilon \not_{z} \not p+\square_{z}\right)\right\} I(-1-\delta, \lambda ; \varepsilon z, p),
\end{aligned}
$$


where $\lambda=d-\frac{3}{2}$ is the anomalous dimension and

$$
\begin{aligned}
I(\kappa, \lambda ; z, p) & =\frac{e^{i p x}}{(2 \pi)^{2}} \int\left(\frac{2}{(x+z-y)^{2}}\right)^{\kappa+1}\left(\frac{2}{(x-y)^{2}}\right)^{\lambda+1} e^{-i p y} d^{4} y \\
= & \frac{e}{\Gamma(\kappa+1) \Gamma(\lambda+1)}\left(\frac{p^{2}}{z^{2}}\right)^{\frac{1}{2}(\kappa+\lambda)} \int_{0}^{1} d u \\
& \cdot\left(\frac{u}{1-u}\right)^{\frac{\kappa-\lambda}{2}} e^{-i u p z \varepsilon} K_{\kappa+\lambda}\left(\sqrt{u(1-u) p^{2} z^{2}}\right)
\end{aligned}
$$

(cf. Eqs. (F.1,2) of Appendix F to [D4]); here $K_{v}$ is the modified Bessel function:

$$
2 K_{v}(\zeta)=\Gamma(v) \Gamma(1-v)\left(I_{-v}(\zeta)-I_{v}(\zeta)\right)
$$

We can use the general formula (A.5) instead of (A.10) to obtain (4.10). Then we need an intermediate analytic regularization $\left(1 / x_{13}^{2} x_{23}^{2}\right) \rightarrow\left(1 / x_{13}^{2} x_{23}^{2}\right)^{1+\delta}$. The operator $\lim _{\delta \rightarrow 0} \frac{d}{d \delta} \delta$ which does not change the initial expressions then recovers a finite part which differs from (4.10) by the value of $\alpha_{0}$. The subtraction term involving the constant $a_{3}$ in (4.7) accounts for this arbitrariness. Similarly, we find using (A.10)

$$
\begin{aligned}
i e \int d \Omega_{z} \int d^{4} y e^{-i p y}\left\langle\bar{\psi}\left(x_{1}+\varepsilon z\right) \gamma_{\mu} \psi\left(x_{1}\right) J_{\gamma}(y)\right\rangle Z_{2}(\varepsilon, \lambda) \\
=-\frac{e^{2} C_{\psi} z(\lambda)}{(2 \pi)^{2}} \frac{\Gamma(2+\lambda)}{\Gamma(1-\lambda)} e^{-i p \cdot x_{1}}\left\{\left[\alpha_{0}^{\prime}+\left(\frac{1+B}{8}+\frac{1}{12}\right) \ln \frac{p^{2} \varepsilon^{2}}{4}\right]\right. \\
\left.\quad \cdot\left(\eta_{\mu \nu} p^{2}-p_{\mu} p_{v}\right)+\eta_{\mu \nu} \frac{2}{\varepsilon^{2}}-\frac{p_{\mu} p_{v}}{6}\right\}+O\left(\varepsilon^{2}\right),
\end{aligned}
$$

where $\alpha_{0}^{\prime}$ is a numerical constant. Comparing (A.12) with (4.9a, b), one determines the constants $\gamma_{i}$ and reproduces the relation (4.11). (The finite transverse part of (A.12) coincides with the second order expression for the photon self-energy.)

Evaluating the contribution of the subtraction terms in (4.9) for $F=A_{\mu}, A_{-}$. $\partial_{\mu} S$, we get the following values for the subtraction constants:

$$
\begin{gathered}
a_{1}=-\frac{2 e^{2} C_{\psi} z(\lambda) \Gamma(2+\lambda)}{(2 \pi)^{2} \Gamma(1-\lambda)}, \quad a_{2}=\frac{a_{1}}{2}\left(\frac{1}{6}+\alpha_{0}\right), \quad a_{3}=\frac{a_{1}}{2} \alpha_{0}, \\
b_{1}=-\frac{e^{2} C_{\psi} z(\lambda)}{(2 \pi)^{2}}(A-\beta+1+2 B) \frac{\Gamma(2+\lambda)}{\Gamma(1-\lambda)}, \\
c_{1}=-\frac{e^{2} C_{\psi} z(\lambda)}{12(2 \pi)^{2}}\left(A-\beta+1-\frac{3}{2}(1+B)-6 \alpha_{0}(1-\beta)\right) \frac{\Gamma(2+\lambda)}{\Gamma(1-\lambda)}, \\
b_{2}=-\frac{e^{2} C_{\psi} z(\lambda) \Gamma(2+\lambda) 2 v(2 \pi)^{2}}{(2 \pi)^{2} \Gamma(1-\lambda) i e}=-6 C_{2} .
\end{gathered}
$$


result

$$
\begin{aligned}
1= & C_{\psi} \lim _{\varepsilon \rightarrow 0} Z_{2}(\varepsilon, \lambda)\left\{p^{2 \lambda}\left[1-\frac{g_{1}+g_{2}}{\lambda} \frac{e^{2}}{(4 \pi)^{2}}\right]\right. \\
& \left.+\left(\frac{4}{\varepsilon^{2}}\right)^{\lambda} \frac{\Gamma(1+\lambda)}{\Gamma(3-\lambda)}\left(2 g_{1}-g_{2}+2 \frac{g_{1}+g_{2}}{\lambda}\right) \frac{e^{2}}{(4 \pi)^{2}}\right\}
\end{aligned}
$$

yields (4.5) and (4.6).

Proceeding now to the implication of the Maxwell equation (4.8) for $F(y)=A_{v}(y)$ or $A_{-}(y)$, we first replace the log-terms in the 3-point functions (3.23), (3.37) by

$$
\log \frac{x_{23}^{2}}{x_{13}^{2}}=\lim _{\delta \rightarrow 0} \frac{1}{\delta}\left\{\left(\frac{2}{x_{13}^{2}}\right)^{\delta}-\left(\frac{2}{x_{23}^{2}}\right)^{\delta}\right\}
$$

and use the general integration formula

$$
\begin{aligned}
& \int d \Omega_{z} z_{\mu} z_{v}(z q)(z p)^{2 \kappa-1} \\
& \quad=\frac{1}{2^{\kappa+2}}\left(\frac{p^{2}}{2}\right)^{\kappa-2}\left[\frac{p^{2}}{2}\left(p q \eta_{\mu \nu}+p_{\mu} q_{\nu}+p_{\nu} q_{\mu}\right)+(\kappa-1) p_{\mu} p_{\nu}(p q)\right] \frac{(2 \kappa) !}{\kappa !(\kappa+2) !} .
\end{aligned}
$$

In studying the small $\varepsilon$ behaviour of

$$
i e Z_{2}(\varepsilon, \lambda) \int d \Omega_{z} \int d^{4} y e^{-i p y}\left\langle\bar{\psi}(x+\varepsilon z) \gamma^{\mu} \psi(x) A_{v}(y)\right\rangle
$$

and representing the Fourier transform of $\frac{x_{13}}{x_{13}^{2}} \gamma^{\mu} \frac{x_{23}}{x_{23}^{2}}$ in (3.37) as

$$
\left(\not x-i \not_{p}\right) \gamma^{\mu}\left(\not x+\varepsilon \not-i \not_{p}\right) \int e^{-i p y} \frac{d^{4} y}{(x-y)^{2}(x+\varepsilon z-y)^{2}},
$$

we need the expansion

$$
K_{0}(\zeta)=-I_{0}(\zeta) \ln \frac{\zeta}{2}+\sum_{k=0}^{\infty} \frac{\psi(k+1)}{(k !)^{2}}\left(\frac{\zeta^{2}}{4}\right)^{k}, \quad \psi(k)=\frac{d}{d k} \ln \Gamma(\kappa),
$$

which gives

$$
\begin{aligned}
I(\kappa,-\kappa ; z, p) & \\
=- & \sum_{s=0}^{\infty} \frac{(-i p z)^{s}}{s !} \sum_{n=0}^{\infty}\left(\frac{p^{2} z^{2}}{4}\right)^{n} \frac{(1+\kappa)_{n+s}(1-\kappa)_{n}}{(n !)^{2}(2 n+s+1) !} \\
& {\left[\ln \frac{p^{2} z^{2}}{2}+\psi(n+\kappa+s+1)+\psi(n+1-\kappa)\right.} \\
- & \psi(2 n+s+2)-2 \psi(n+1)],
\end{aligned}
$$

where $(a)_{n}=\frac{\Gamma(a+n)}{\Gamma(a)}$. A straightforward (though rather lengthy) computation leads to (4.10) with

$$
\alpha_{0}=\frac{1+B}{8}\left(-\frac{1}{2}-\psi(1)\right)-\frac{1}{6}\left(\psi(1)+\frac{5}{3}\right) .
$$


We can use the general formula (A.5) instead of (A.10) to obtain (4.10). Then we need an intermediate analytic regularization $\left(1 / x_{13}^{2} x_{23}^{2}\right) \rightarrow\left(1 / x_{13}^{2} x_{23}^{2}\right)^{1+\delta}$. The operator $\lim _{\delta \rightarrow 0} \frac{d}{d \delta} \delta$ which does not change the initial expressions then recovers a finite part which differs from (4.10) by the value of $\alpha_{0}$. The subtraction term involving the constant $a_{3}$ in (4.7) accounts for this arbitrariness. Similarly, we find using (A.10)

$$
\begin{array}{rl}
i e \int d & d \Omega_{z} \int d^{4} y e^{-i p y}\left\langle\bar{\psi}\left(x_{1}+\varepsilon z\right) \gamma_{\mu} \psi\left(x_{1}\right) J_{\gamma}(y)\right\rangle Z_{2}(\varepsilon, \lambda) \\
= & -\frac{e^{2} C_{\psi} z(\lambda)}{(2 \pi)^{2}} \frac{\Gamma(2+\lambda)}{\Gamma(1-\lambda)} e^{-i p \cdot x_{1}}\left\{\left[\alpha_{0}^{\prime}+\left(\frac{1+B}{8}+\frac{1}{12}\right) \ln \frac{p^{2} \varepsilon^{2}}{4}\right]\right. \\
& \left.\cdot\left(\eta_{\mu v} p^{2}-p_{\mu} p_{v}\right)+\eta_{\mu v} \frac{2}{\varepsilon^{2}}-\frac{p_{\mu} p_{v}}{6}\right\}+O\left(\varepsilon^{2}\right),
\end{array}
$$

where $\alpha_{0}^{\prime}$ is a numerical constant. Comparing (A.12) with (4.9a, b), one determines the constants $\gamma_{i}$ and reproduces the relation (4.11). (The finite transverse part of (A.12) coincides with the second order expression for the photon self-energy.)

Evaluating the contribution of the subtraction terms in (4.9) for $F=A_{\mu}, A_{-}$. $\partial_{\mu} S$, we get the following values for the subtraction constants:

$$
\begin{gathered}
a_{1}=-\frac{2 e^{2} C_{\psi} z(\lambda) \Gamma(2+\lambda)}{(2 \pi)^{2} \Gamma(1-\lambda)}, \quad a_{2}=\frac{a_{1}}{2}\left(\frac{1}{6}+\alpha_{0}\right), \quad a_{3}=\frac{a_{1}}{2} \alpha_{0}, \\
b_{1}=-\frac{e^{2} C_{\psi} z(\lambda)}{(2 \pi)^{2}}(A-\beta+1+2 B) \frac{\Gamma(2+\lambda)}{\Gamma(1-\lambda)}, \\
c_{1}=-\frac{e^{2} C_{\psi} z(\lambda)}{12(2 \pi)^{2}}\left(A-\beta+1-\frac{3}{2}(1+B)-6 \alpha_{0}(1-\beta)\right) \frac{\Gamma(2+\lambda)}{\Gamma(1-\lambda)}, \\
b_{2}=-\frac{e^{2} C_{\psi} z(\lambda) \Gamma(2+\lambda) 2 v(2 \pi)^{2}}{(2 \pi)^{2} \Gamma(1-\lambda) i e}=-6 C_{2} .
\end{gathered}
$$

In dealing with 4-point functions, we only work in $x$-space and use in addition to (A.6) the relation (A.9).

Acknowledgements. We would like to thank Dr. V. Dobrev and Dr. A. Ganchev for making available their unpublished calculations and Dr. E. Nissimov for valuable discussions.

Note added in proof. Equation (2.21) [or (4.1d)] is consistent with the commutation relations (3.32), (3.35). To amend the situation we should add a trilinear term, $\frac{q}{4} A_{-}^{2} \square^{2} S$, replacing the Lagrangian (2.19), (2.14) by

$$
\begin{aligned}
L= & \frac{1}{2} F^{\mu v}\left(\frac{1}{2} F_{\mu v}-\partial_{\mu} A_{v}+\partial_{v} A_{\mu}\right)-\frac{q}{2} A_{-} \square \partial A+\frac{\beta}{8}\left(\square A_{-}\right)^{2}+\frac{v}{2}(\square S)^{2} \\
& -\bar{\psi}\left[\not \partial-i e\left(A-A_{-} \not \partial S\right)\right] \psi+\frac{q}{4} A_{-}^{2} \square^{2} S .
\end{aligned}
$$


The Maxwell and the Dirac equations (2.7) and (2.20) remain unchanged while the gauge fixing equations for $\partial A$ and $S$ assume the form

$$
\frac{q}{2} \square \partial A+\partial_{\mu} S J^{\mu}=\frac{q}{2} A_{-} \square^{2} S ; \quad v \square^{2} S+\partial_{\mu} A_{-} J^{\mu}+\frac{q}{4} \square^{2} A_{-}^{2}=0 .
$$

The new set of equations has the virtue of being invariant with respect to the 1-parameter family of field transformations

$$
A_{-} \rightarrow A_{-}+\lambda, \quad A_{\mu} \rightarrow A_{\mu}+\lambda \partial_{\mu} S .
$$

It is straightforward to verify [using (3.7) and (3.23a)] that

$$
\begin{aligned}
\frac{1}{4} \square_{3}^{2}\left\langle\psi(1) \bar{\psi}(2) A_{-}^{2}(3)\right\rangle_{0} & =\frac{e^{2}}{8 \pi^{4}}\left[\frac{x_{12}^{2}}{\left(x_{13}^{2}+i o x_{13}^{0}\right)\left(x_{23}^{2}+i o x_{23}^{0}\right)}\right]^{2}\langle\psi(1) \bar{\psi}(2)\rangle_{0} \\
& =-\left\langle\psi(1) \bar{\psi}(2) J^{\mu}(3) \partial_{\mu} A_{-}(3)\right\rangle_{0}
\end{aligned}
$$

so that [according to (2)] $v \square_{3}^{2}\langle\psi(1) \bar{\psi}(2) S(3)\rangle_{0}=0$ in agreement with (3.36).

The argument of Sect. $2 \mathrm{C}$ is not directly applicable to this modified Lagrangian, however a calculation based on a power series expansion in the new trilinear term indicates that it does not affect gauge invariant matrix elements.

\section{References}

[A1] Adler, S.: Short distance behaviour of quantum electrodynamics and an eigenvalue condition for $\alpha$, Phys. Rev. D5, 3021-3047 (1972), Erratum, ibid. D7, 1948 (1973); Euclidean quantum electrodynamics on the 5-dimensional unit hypersphere. Phys. Rev. D6, 3445-3461 (1972), Erratum, ibid. D7, 3821 (1973)

[A2] Adler, S., Bardeen, W.: Quantum electrodynamics without photon self-energy parts: an application of the Callan-Symanzik scaling equations. Phys. Rev. D4, 3045-3054 (1971) Erratum, ibid. D6, 734 (1972)

[A3] Aizenman, M.: Geometric analysis of $\varphi^{4}$ fields and Ising models. Commun. Math. Phys. 86, 1-40 (1982)

[B1] Baker, M., Johnson, K: Applications of conformal symmetry in quantum electrodynamics. Physica 96A, 120-130 (1979)

[B2] Bateman, H.: The transformation of the electrodynamical equations. Proc. London Math. Soc. 8, 223-264 (1910)

[B3] Bayen, F., Flato, M.: (a) Remarks on conformal space. J. Math. Phys. 17, 1112-1114 (1976); (b) Conformal covariant QED revisited (to be published)

[B4] Binegar, B., Fronsdal, C., Heidenreich, W.: Conformal QED. J. Math. Phys. 24, 2828-2846 (1983)

[B5] Brandt, R.: Gauge invariance in quantum electrodynamics. Ann. Phys. (N.Y.) 52, 122-176 (1969)

[C1] Cunningham, E.: The principle of relativity in electrodynamics and an extension thereof. Proc. London Math. Soc. 8, 77-98 (1909)

[D1] Dell'Antonio, G.F.: On dilation invariance and the Wilson expansion. Nuovo Cim. 12A, 756-762 (1972)

[D2] Dirac, P.A.M.: Wave equations in conformal space. Ann. Math. 37, 429-442 (1936)

[D3] Dobrev, V.K., Petkova, V.B., Petrova, S.G., Todorov, I.T.: Dynamical derivation of vacuum operator product expansion in Euclidean conformal quantum field theory. Phys. Rev. D13, 887-912 (1976)

[D4] Dobrev, V.K., Mack, G., Petkova, V.B., Petrova, S.G., Todorov, I.T.: Harmonic analysis. In: Lecture Notes in Physics, Vol. 63. Berlin, Heidelberg, New York: Springer 1977 
[D5] Dobrev, V.K., Ganchev, A., Petkova, V.B.: Schwinger-Dyson equations in conformal quantum electrodynamics (1981) (unpublished)

[E1] Englert, F.: Self-consistent approach to the fine-structure constant. Nuovo Cim. 16A, 557572 (1973)

[F1] Ferrara, S., Gatto, R., Grillo, A.: Logarithmic scaling and spontaneous breaking. Phys. Lett. 42B, 264-266 (1972)

[F2] Ferrari, R., Picasso, L., Strocchi, F.: Some remarks on local operators in quantum electrodynamics. Commun. Math. Phys. 35, 25-38 (1974)

[F3] Fradkin, E.S., Kozhevnikov, A.A., Palchik, M.Ya., Pomeransky, A.A.: Conformal invariant formulation of quantum electrodynamics. Commun. Math. Phys. 91, 529-541 (1983)

[F4] Fradkin, E.S., Palchik, M.Ya.: Recent developments in conformal invariant QFT. Phys. Rep. 44C, 249-349 (1978)

[F5] Fröhlich, J.: On the triviality of $\lambda \varphi_{d}^{4}$ theories and the approach to the critical point in $d \geq 4$ dimensions. Nucl. Phys. B200, 281-296 (1982)

[F6] Fry, M.: Connection between the electron propagator and the Baker-Johnson function in conformal invariant quantum electrodynamics. Nuovo Cim. 31A, 129-150 (1976)

[F7] Furlan, P., Petkova, V.B., Sotkov, G.M., Todorov, I.T.: Conformal quantum electrodynamics with a 5-potential, ISAS preprint 52/83/EP, Trieste; Conformal quantum electrodynamics and non-decomposable representations (to be submitted to Rivista Nuovo Cim.) ISAS preprint 51/84/EP, Trieste

[J1] Johnson, K., Willey, R., Baker, M.: Vacuum polarization in quantum electrodynamics. Phys. Rev. 163, 1699-1715 (1967)

[K1] Krasnikov, N.V.: Is finite renormalization of electric charge possible? Phys. Lett. 126B, 483-484 (1983)

[M1] Mack, G., Salam, Abdus: Finite component field representations of the conformal group. Ann. Phys. (N.Y.) 53, 174-202 (1969)

[M2] Mack, G., Symanzik, K.: Currents, stress tensor and generalized unitarity in conformal invariant quantum field theory. Commun. Math. Phys. 27, 247-281 (1972)

[M3] Mack, G., Todorov, I.T.: Conformal invariant Green functions without ultraviolet divergences. Phys. Rev. D8, 1787 (1973)

[M4] Mannheim, P.: Structure of the vertex function in finite quantum electrodynamics. Preprint LBL-3610, Berkeley (1974)

[M5] Matsubara, Y., Suzuki, T., Yotsuyanagi, I.: On a possible inconsistency of the $\varphi^{4}$ and the Yukawa theories in four dimensions, Kanazawa Univ. Preprint DPKU-8303 (1983)

[M6] Matsubara, Y., Suzuki, T., Yotsuyanagi, I.: Can (QED) 4 be a trivial theory? Kanazawa Univ. Preprint DPKU-8304 (1983)

[M7] Mayer, D.H.: Vector and tensor fields in conformal space. J. Math. Phys. 16, 884-893 (1975)

[P1] Palchik, M.Ya.: On the operator product $j_{\mu}(x) \psi(x)$ in the Thirring model. Short Communications of the Lebedev Physics Institute, No.4, 19-24 (1980) (in Russian)

[P2] Palchik, M.Ya.: A new approach to the conformal invariance problem in quantum electrodynamics. J. Phys. A16, 1523-1527 (1983)

[P3] Petkova, V.B., Sotkov, G.M.: Exceptional representations of the conformal group and applications, I and II. Bulg. J. Phys. 10, 144-172, 256-277 (1983); The six-point families of exceptional representations of the conformal group, ISAS preprint 15/83/E.P. Trieste; Lett. Math. Phys. 8, 217-226 (1984)

[P4] Popov, V.N.: Path Integrals in Quantum Field Theory and Quantum Statistical Mechanics (Atomizdat, Moscow 1976; in Russian). See, in particular, Chap. 3

[S1] Salam, Abdus, Strathdee, J.: Nonlinear realizations. II. Conformal symmetry. Phys. Rev. 184, 1760-1768 (1969)

[S2] Schroer, B.: A necessary and sufficient condition for the softness of the trace of the energymomentum tensor. Lett. Nuovo Cim. 2, 867-872 (1972) 
[S3] Sotkov, G., Stoyanov, D.: On the conformal invariance in quantum electrodynamics. J. Phys. A13, 2807-2816 (1980); Conformal quantization of electrodynamics. J. Phys. A16, 2817-2826 (1983)

[S4] Strocchi, F.: Extension of Jost and Schroer's theorem to quantum electrodynamics. Phys. Rev. D6, 1193-1195 (1972)

[S5] Strocchi, F., Wightman, A.S.: Proof of the charge superselection rule in local relativistic quantum field theory. J. Math. Phys. 15, 2198-2224 (1974); Erratum, ibid. 17, 1930-1931 (1976)

[S6] Symanzik, K.: Green's functions method and renormalization of renormalizable quantum field theory. In: Lectures in High Energy Physics, Jaksič, B. (ed.). Zagreb 1961. New York: Gordon and Breach, 1965; Euclidean quantum field theory. In: Coral Gables Conference on Fundamental Interactions at High Energy, Gudehus, T. et al. (eds.). New York: Gordon and Breach, 1969, pp. 19-31

[T1] Todorov, I.T., Mintchev, M.C., Petkova, V.B.: Conformal Invariance in Quantum Field Theory. Scuola Normale Superiore, Pisa 1978

[T2] Todorov, I.T.: Local field representations of the conformal group and their applications. ZiF-Bielefeld preprint (1984)

[Z1] Zaikov, R.P.:On conformal invariance in gauge theories; quantum electrodynamics. JINR preprint E2-83-28 Dubna (1983)

[Z2] Zavialov, O.I.: Renormalized Feynman Diagrams. Moscow: Nauka, 1979; in Russian

Communicated by G. Mack

Received July 11, 1984; in revised form August 28, 1984 
